\title{
An Efficient Approach to Achieve Compositionality using Optimized Multi-Version Object Based Transactional Systems ${ }^{1}$
}

\author{
Chirag Juyal $^{1}$, Sandeep Kulkarni ${ }^{2}$, Sweta Kumari ${ }^{1}$, Sathya Peri ${ }^{1}$ and Archit Somani ${ }^{12}$ \\ Department of Computer Science \& Engineering, IIT Hyderabad, India ${ }^{1}$ \\ (cs17mtech11014, cs15resch01004, sathya-p, cs15resch01001) eiith.ac.in \\ Department of Computer Science, Michigan State University, MI, USA ${ }^{2}$ \\ sandeep@cse.msu.edu
}

\begin{abstract}
In the modern era of multi-core systems, the main aim is to utilize the cores properly. This utilization can be done by concurrent programming. But developing a flawless and well-organized concurrent program is difficult. Software Transactional Memory Systems (STMs) are a convenient programming interface which assist the programmer to access the shared memory concurrently without worrying about consistency issues such as priority-inversion, deadlock, livelock, etc. Another important feature that STMs facilitate is compositionality of concurrent programs with great ease. It composes different concurrent operations in a single atomic unit by encapsulating them in a transaction.
\end{abstract}

Many STMs available in the literature execute read/write primitive operations on memory buffers. We represent them as Read-Write STMS or RWSTMs. Whereas, there exist some STMs (transactional boosting and its variants) which work on higher level operations such as insert, delete, lookup, etc. on a hash-table. We refer these STMs as Object Based STMs or OSTMs.

The literature of databases and RWSTMs say that maintaining multiple versions

\footnotetext{
${ }^{1}$ A preliminary version of this paper appeared in 20th International Symposium on Stabilization, Safety, and Security of Distributed Systems (SSS 2018) and awarded with the Best Student Paper Award. A poster version of this work received Best Poster Award in NETYS 2018.

${ }^{2}$ Author sequence follows the lexical order of last names. All the authors can be contacted at the addresses given above. Archit Somani's phone number: +91 - 7095044601.
} 
ensures greater concurrency. This motivates us to maintain multiple version at higher level with object semantics and achieves greater concurrency. So, this paper proposes the notion of Optimized Multi-version Object Based STMs or OPT-MVOSTMs which encapsulates the idea of multiple versions in OSTMs to harness the greater concurrency efficiently. For efficient memory utilization, we develop two variations of OPT-MVOSTMs. First, OPT-MVOSTM with garbage collection (or OPT-MVOSTM-GC) which uses unbounded versions but performs garbage collection scheme to delete the unwanted versions. Second, finite version OPT-MVOSTM (or OPT-KOSTM) which maintains at most $K$ versions by replacing the oldest version when $(K+1)^{t h}$ version is created by the current transaction.

We propose the OPT-MVOSTMs for hash-table and list objects as OPT-HT-MVOSTM and $O P T$-list-MVOSTM respectively. For memory utilization, we propose two variants of both the algorithms as OPT-HT-MVOSTM-GC, OPT-HT-KOSTM and OPT-listMVOSTM-GC, OPT-list-KOSTM respectively. OPT-HT-KOSTM performs best among its variants and outperforms state-of-the-art hash-table based STMs (HT-OSTM, ESTM, RWSTM, HT-MVTO, HT-KSTM) by a factor of 3.62, 3.95, 3.44, 2.75, 1.85 for workload W1 (90\% lookup, 8\% insert and 2\% delete), 1.44, 2.36, 4.45, 9.84, 7.42 for workload W2 (50\% lookup, 25\% insert and 25\% delete), and 2.11, 4.05, 7.84, 12.94, 10.70 for workload W3 (10\% lookup, $45 \%$ insert and 45\% delete) respectively. Similarly, OPT-list-KOSTM performs best among its variants and outperforms state-of-the-art list based STMs (list-OSTM, Trans-list, Boosting-list, NOrec-list, list-MVTO, list-KSTM) by a factor of 2.56, 25.38, 23.57, 27.44, 13.34, 5.99 for W1, 1.51, 20.54, 24.27, 29.45, $24.89,19.78$ for $\mathrm{W} 2$, and $2.91,32.88,28.45,40.89,173.92,124.89$ for $\mathrm{W} 3$ respectively. OPT-MVOSTMs are generic for other data structures as well. We rigorously proved that OPT-MVOSTMs satisfy opacity and ensure that transaction with lookup only methods will never return abort while maintaining unbounded versions.

Keywords: Software Transactional Memory Systems, Optimized, Lazyrb-list, Hash-Table, List, Object, Multi-version, Compositionality, Opacity, Keys 


\section{Introduction}

Nowadays, multi-core systems are in trend which necessitated the need for concurrent programming to exploit the cores appropriately. Howbeit, developing the correct and efficient concurrent programs is difficult. Software Transactional Memory Systems (STMs) are a convenient programming interface which assist the programmer to access the shared memory concurrently using multiple threads without worrying about consistency issues such as deadlock, livelock, priority-inversion, etc. STMs facilitate one more feature compositionality of concurrent programs with great ease which makes it more approachable. Different concurrent operations that need to be composed to form a single atomic unit is achieved by encapsulating them in a transaction. In this paper, we discuss various STMs such as read-write STMs (or RWSTMs), object based STMs (or OSTMs) available in the literature along with the benefits of OSTMs over RWSTMs. After that, we motivated from multi-version RWSTMs and propose multi-version object based STMs (or MVOSTMs) [1] which maintain multiple versions and improves the concurrency further. Later, we made a couple of modifications (discussed in Section 4 Section 5 and Section 7) to optimize the MVOSTMs and propose optimized MVOSTMs (or OPT-MVOSTMS).

Read-Write STMs: There exists a lot of popular STMs in the literature such as ESTM [2], NOrec [3] which executes read/write operations on transaction objects or t-objects. We represent these STMs as Read-Write STMs or RWSTMs. RWSTMs typically export following methods: (1) t_begin: which begins a transaction with a unique identity, (2) $t_{-}$read (or $r$ ): which reads the value of t-object from shared memory, (3) t_write (or $w$ ): which writes the new value to t-object in its local memory, (4) tryC: which validates the values written to t-objects by the transaction and tries to commit. If all the updates made by the transaction is consistent then updates reflect to the shared memory and transaction returns commit, and (5) tryA: which returns abort on any inconsistency.

Object based STMs: There are few STMs available in the literature which executes higher level operations such as insert, delete, lookup on hash-table. We represent these STMs as Object based STMs or OSTMs. The concept of Boosting by Herlihy et al. [4], the optimistic variant by Hassan et al. [5] and recently HT-OSTM system by Peri et al. 


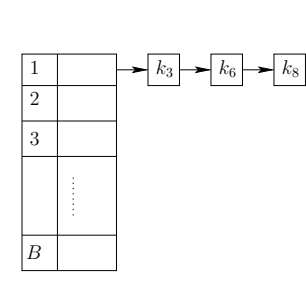

(a) Underlying hash-table

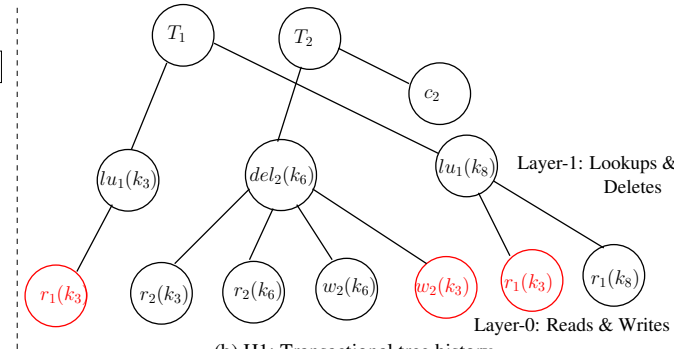

(b) H1: Transactional tree history

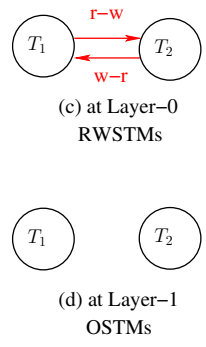

OSTMs

Figure 1: Advantages of OSTMs over RWSTMs

[6] are some examples that demonstrate the performance benefits achieved by OSTMs.

Peri et al. [6] showed that OSTMs provide greater concurrency than RWSTMs while reducing the number of aborts.

Benefits of OSTMs over RWSTMs: To show the benefits of OSTMs, We consider a hash-table based STM system which invokes insert (or ins), lookup (or $l u$ ) and delete (or $d e l$ ) method. Each hash-table consists of $B$ buckets with the elements in each bucket arranged in the form of a linked-list. Figure 1 (a) represents a hash-table with the first bucket containing keys $\left\langle k_{3}, k_{6}, k_{8}\right\rangle$. Figure 1 (b) shows the execution by two transaction $T_{1}$ and $T_{2}$ represented in the form of a tree. $T_{1}$ performs lookup operations on keys $k_{3}$ and $k_{8}$ while $T_{2}$ performs a delete on $k_{6}$. The delete on key $k_{6}$ generates read on the keys $k_{3}, k_{6}$ and writes the keys $k_{6}, k_{3}$ assuming that delete is performed similar to delete operation in lazy-list [7]. The lookup on $k_{3}$ generates read on $k_{3}$ while the lookup on $k_{8}$ generates read on $k_{3}, k_{8}$. Note that in this execution $k_{6}$ has already been deleted by the time lookup on $k_{8}$ is performed.

In this execution, we denote the read-write operations (leaves) as layer- 0 and $l u, d e l$ methods as layer-1. Consider the history (execution) at layer-0 (while ignoring higherlevel operations), denoted as $H 0$. It can be verified this history is not opaque [8]. This is because, between the two reads of $k_{3}$ by $T_{1}, T_{2}$ writes to $k_{3}$. It can be seen that if history $H 0$ is input to an RWSTMs one of the transactions between $T_{1}$ or $T_{2}$ would be aborted to ensure opacity [8]. Figure 1](c) shows the presence of a cycle in the conflict graph of $H 0$.

Now, consider the history $H 1$ at layer- 1 consists of $l u$, and del methods, while 


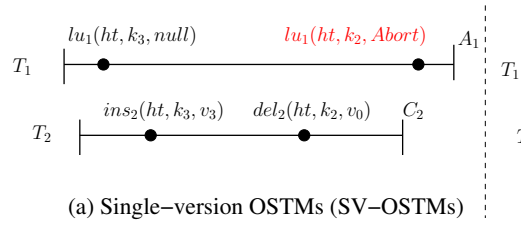

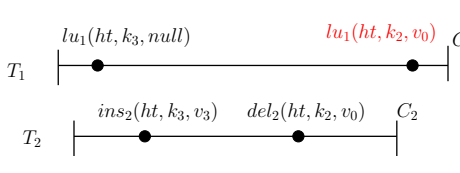

(b) Optimized Multi-version OSTMs

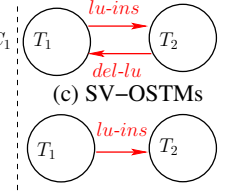

(d) OPT-MVOSTMs

Figure 2: Advantages of optimized multi-version over single version OSTM

ignoring the read/write operations since they do not overlap (referred to as pruning in [9, Chap 6]). These methods work on distinct keys $\left(k_{3}, k_{6}\right.$, and $\left.k_{8}\right)$. They do not overlap and are not conflicting. So, they can be re-ordered in either way. Thus, $H 1$ is opaque [8] with equivalent serial history $T_{1} T_{2}$ (or $T_{2} T_{1}$ ) and the corresponding conflict graph shown in Figure 1 (d). Hence, a hash-table based OSTM system does not abort any of $T_{1}$ or $T_{2}$. This shows that $O S T M \mathrm{~s}$ can reduce the number of aborts and provide greater concurrency.

Multi-Version Object Based STMs: Some of the OSTMs such as [4], [5], [6] exploits the advantages of it. In this paper, we propose and analyze Optimized Multi-version Object Based STMs or OPT-MVOSTMs along with the rigorous correctness proof. This work is motivated by the observation that databases and RWSTMs achieves greater concurrency by storing multiple versions corresponding to each t-object [10]. Specifically, maintaining multiple versions can ensure that more read operations succeed because the reading operation will obtain an appropriate version to read. Our goal is to analyze the benefit of OPT-MVOSTMs over both single version OSTMs and multi-version RWSTMs. The potential benefit of OPT-MVOSTMS over OSTMs and multi-version RWSTMs: We now illustrate the advantage of OPT-MVOSTMs as compared to single-version OSTMs (SV-OSTMs) using the hash-table object with $B$ buckets having the same operations as discussed above: ins, lu, del. Figure 2 (a) represents a history $\mathrm{H}$ with two concurrent transactions $T_{1}$ and $T_{2}$ operating on a hash-table $h t . T_{1}$ first tries to perform a $l u$ on key $k_{3}$. But due to the absence of key $k_{3}$ in $h t$, it obtains a value of null. Then $T_{2}$ invokes ins method on the same key $k_{3}$ and inserts the value $v_{3}$ in $h t$. Then $T_{2}$ deletes the key $k_{2}$ from $h t$ and returns $v_{0}$ implying that some other transaction had previously inserted $v_{0}$ into $k_{2}$. The second method of $T_{1}$ is $l u$ on the key $k_{2}$. With this execution, any $S V$-OSTM system has to return abort for $T_{1}$ 's $l u$ operation to ensure 
correctness, i.e., opacity. Otherwise, if $T_{1}$ would have obtained a return value $v_{0}$ for $k_{2}$, then the history would not be opaque anymore. This is reflected by a cycle in the corresponding conflict graph between $T_{1}$ and $T_{2}$, as shown in Figure 2 (c). Thus to ensure opacity, $S V$-OSTM system has to return abort for $T_{1}$ 's lookup on $k_{2}$.

In an OPT-MVOSTMs based on hash-table, denoted as OPT-HT-MVOSTM, whenever a transaction inserts or deletes a key $k$, a new version is created. Consider the above example with an OPT-HT-MVOSTM, as shown in Figure 2(b). Even after $T_{2}$ deletes $k_{2}$, the previous value of $v_{0}$ is still retained. Thus, when $T_{1}$ invokes $l u$ on $k_{2}$ after the delete on $k_{2}$ by $T_{2}, O P T-H T-M V O S T M$ return $v_{0}$ (as previous value). With this, the resulting history is opaque with equivalent serial history being $T_{1} T_{2}$. The corresponding conflict graph is shown in Figure 2(d) does not have a cycle.

Thus, OPT-MVOSTM reduces the number of aborts and achieve greater concurrency than $S V$-OSTMs while ensuring the compositionality. We believe that the benefit of OPT-MVOSTM over multi-version RWSTM is similar to SV-OSTM over single-version RWSTM as explained above. OPT-MVOSTM is a generic concept which can be applied to any data structure. In this paper, we have considered the hash-table and list based OPT-MVOSTMs as OPT-HT-MVOSTM and OPT-list-MVOSTM respectively. If the bucket size $B$ of hash-table becomes 1 then hash-table based OPT-MVOSTMs boils down to the list based OPT-MVOSTMs.

OPT-HT-MVOSTM and OPT-list-MVOSTM use an unbounded number of versions for each key. To address this issue, we develop two variants for both hash-table and list data structures (or DS): (1) A garbage collection method in OPT-MVOSTMs to delete the unwanted versions of a key, denoted as OPT-MVOSTM-GC. Garbage collection gave an average performance gain of $16 \%$ over OPT-MVOSTM without garbage collection in the best case. Thus, the overhead of garbage collection scheme is less than the performance improvement due to improved memory usage. (2) Placing a limit of $K$ on the number versions in OPT-MVOSTM, resulting in OPT-KOSTM. This gave an average performance gain of $24 \%$ over OPT-MVOSTM without garbage collection in the best case.

Experimental results show that OPT-HT-KOSTM performs best among its variants and outperforms state-of-the-art hash-table based STMs (HT-OSTM, ESTM, RWSTM, 
HT-MVTO, HT-KSTM) by a factor of 3.62, 3.95, 3.44, 2.75, 1.85 for workload W1 (90\% lookup, 8\% insert and 2\% delete), 1.44, 2.36, 4.45, 9.84, 7.42 for workload W2 (50\% lookup, 25\% insert and 25\% delete), and 2.11, 4.05, 7.84, 12.94, 10.70 for workload W3 (10\% lookup, 45\% insert and 45\% delete) respectively. Similarly, OPT-list-KOSTM performs best among its variants and outperforms state-of-the-art list based STMs (list-OSTM, Trans-list, Boosting-list, NOrec-list, list-MVTO, list-KSTM) by a factor of 2.56, 25.38, 23.57, 27.44, 13.34, 5.99 for $\mathrm{W} 1,1.51,20.54,24.27,29.45$, 24.89, 19.78 for $\mathrm{W} 2$, and 2.91, 32.88, 28.45, 40.89, 173.92, 124.89 for W3 respectively. To the best of our knowledge, this is the first work to explore the idea of using multiple versions in OSTMs to achieve greater concurrency.

\section{Contributions of the paper:}

- We propose a new notion of optimized multi-version objects based STM system as OPT-MVOSTM in Section 4. In this paper, we develop it for list and hashtable objects as OPT-list-MVOSTM and OPT-HT-MVOSTM respectively. OPTMVOSTM is generic for other data structures as well.

- For efficient space utilization in OPT-MVOSTMs with unbounded versions, we develop Garbage Collection for OPT-MVOSTM (i.e. OPT-MVOSTM-GC) and bounded version OPT-MVOSTM (i.e. OPT-KOSTM).

- Section 6 shows that OPT-list-MVOSTM and OPT-HT-MVOSTM satisfy standard correctness-criterion of STMs, opacity [8].

- Experimental analysis of both OPT-list-MVOSTM and OPT-HT-MVOSTM with state-of-the-art STMs are present in Section 7. Proposed OPT-list-MVOSTM and OPT-HT-MVOSTM provide greater concurrency and reduces the number of aborts as compared to MVOSTMs, SV-OSTMs, single-version RWSTMs and, multi-version $R W S T M$ s while maintaining multiple versions corresponding to each key.

Roadmap: The paper is organized as follows. We describe our building system model in Section 2, In Section 3, we formally define the graph characterization of opacity. Section 4 represents the OPT-MVOSTMs design and data structure. Section 5 shows the 
working of OPT-HT-MVOSTMs and its algorithms. We formally prove the correctness of OPT-MVOSTMs in Section 6 In Section 7 we show the experimental evaluation of OPT-MVOSTMs with state-of-art-STMs. Finally, we conclude in Section 8.

\section{Building System Model}

Our assumption follows [11, 6] in which the system consists of a finite set of $p$ processes, $p_{1}, \ldots, p_{n}$, accessed by a finite number of $n$ threads in a completely asynchronous fashion and communicates each other using shared keys (or objects). The threads invoke higher level methods on the shared objects and get corresponding responses. Consequently, we make no assumption about the relative speeds of the threads. We also assume that none of these processors and threads fail or crash abruptly.

Events and Methods: We assume that the threads execute atomic events and the events by different threads are (1) read/write on shared/local memory objects, (2) method invocations (or $i n v$ ) event and responses (or $r s p$ ) event on higher level shared memory objects.

Within a transaction, a process can invoke layer-1 methods (or operations) on a hash-table t-object. A hash-table $(h t)$ consists of multiple key-value pairs of the form $\langle k, v\rangle$. The keys and values are respectively from sets $\mathscr{K}$ and $\mathscr{V}$. The methods that a thread can invoke are: (1) t_begin $_{i}()$ : begins a transaction and returns a unique id to the invoking thread. (2) $t_{-}$insert $_{i}(h t, k, v)$ : transaction $T_{i}$ inserts a value $v$ onto key $k$ in $h t$. (3) $t_{-} \operatorname{delete}_{i}(h t, k, v)$ : transaction $T_{i}$ deletes the key $k$ from the hash-table $h t$ and returns the current value $v$ for $T_{i}$. If key $k$ does not exist, it returns null. (4) $t_{\_}$lookup $_{i}(h t, k, v)$ : returns the current value $v$ for key $k$ in $h t$ for $T_{i}$. Similar to $t$ delete, if the key $k$ does not exist then $t_{-} l o o k u p$ returns null. (5) try $C_{i}()$ : which tries to commit all the operations of $T_{i}$ and (6) $\operatorname{try} A_{i}()$ : aborts $T_{i}$. We assume that each method consists of an $i n v$ and $r s p$ event.

We denote $t_{-}$insert and t_delete as update methods (or upd_method or up) since both of these change the underlying data structure. We denote t_delete and t_lookup as return-value methods (or rv_method or rvm) as these operations return values from $h t$. A method may return $o k$ if successful or $\mathscr{A}$ (abort) if it sees an inconsistent state of $h t$. 
Formally, we denote a method $m$ by the tuple $\left\langle e v t s(m),<_{m}\right\rangle$. Here, evts $(m)$ are all the events invoked by $m$ and the $<_{m}$ a total order among these events.

Transactions: Following the notations used in database multi-level transactions[9], we model a transaction as a two-level tree. The layer- 0 consist of read/write events and layer-1 of the tree consists of methods invoked by a transaction.

Having informally explained a transaction, we formally define a transaction $T$ as the tuple $\left\langle\operatorname{evts}(T),<_{T}\right\rangle$. Here $\operatorname{evts}(T)$ are all the read/write events at layer- 0 of the transaction. $<_{T}$ is a total order among all the events of the transaction.

We denote the first and last events of a transaction $T_{i}$ as $T_{i}$.firstEvt and $T_{i} . l a s t E v t$. Given any other read/write event $r w$ in $T_{i}$, we assume that $T_{i}$. firstEvt $<_{T_{i}} r w<_{T_{i}}$ $T_{i}$.lastEvt. All the methods of $T_{i}$ are denoted as $\operatorname{methods}\left(T_{i}\right)$. We assume that for any method $m$ in methods $\left(T_{i}\right)$,evts $(m)$ is a subset of evts $\left(T_{i}\right)$ and $<_{m}$ is a subset of $<_{T_{i}}$. We assume that if a transaction has invoked a method, then it does not invoke a new method until it gets the response of the previous one. Thus all the methods of a transaction can be ordered by $<_{T_{i}}$. Formally, $\left(\forall m_{p}, m_{q} \in \operatorname{methods}\left(T_{i}\right):\left(m_{p}<_{T_{i}}\right.\right.$ $\left.\left.\left.m_{q}\right) \vee\left(m_{q}<_{T_{i}} m_{p}\right)\right)\right\rangle$, here $m_{p}$ and $m_{q}$ are $p_{t h}$ and $q_{t h}$ methods of $T_{i}$ respectively.

Histories: A history is a sequence of events belonging to different transactions. The collection of events is denoted as evts $(H)$. Similar to a transaction, we denote a history $H$ as tuple $\left\langle\operatorname{evts}(H),<_{H}\right\rangle$ where all the events are totally ordered by $<_{H}$. The set of methods that are in $H$ is denoted by methods $(H)$. A method $m$ is incomplete if $\operatorname{inv}(m)$ is in evts $(H)$ but not its corresponding response event. Otherwise, $m$ is complete in $H$.

Coming to transactions in $H$, the set of transactions in $H$ are denoted as $\operatorname{txns}(H)$. The set of committed (resp., aborted) transactions in $H$ is denoted by committed $(H)$ (resp., aborted $(H)$ ). The set of live transactions in $H$ are those which are neither committed nor aborted and denoted as live $(H)=\operatorname{txns}(H)-\operatorname{committed}(H)-$ aborted $(H)$. On the other hand, the set of terminated transactions are those which have either committed or aborted and is denoted by $\operatorname{term}(H)=\operatorname{committed}(H) \cup$ aborted $(H)$.

The relation between the events of transactions \& histories is analogous to the relation between methods $\&$ transactions. We assume that for any transaction $T$ in 
$\operatorname{txns}(H), \operatorname{evts}(T)$ is a subset of $\operatorname{evts}(H)$ and $<_{T}$ is a subset of $<_{H}$. Formally, $\langle\forall T \in$ $\left.\operatorname{txns}(H):(\operatorname{evts}(T) \subseteq \operatorname{evts}(H)) \wedge\left(<_{T} \subseteq<_{H}\right)\right\rangle$.

We denote two histories $H_{1}, H_{2}$ as equivalent if their events are the same, i.e., $\operatorname{evts}\left(H_{1}\right)=\operatorname{evts}\left(H_{2}\right)$. A history $H$ is qualified to be well-formed if: (1) all the methods of a transaction $T_{i}$ in $H$ are totally ordered, i.e. a transaction invokes a method only after it receives a response of the previous method invoked by it (2) $T_{i}$ does not invoke any other method after it received an $\mathscr{A}$ response or after $\operatorname{try} C(o k)$ method. We only consider well-formed histories for OPT-MVOSTM.

A method $m_{i j}\left(j^{\text {th }}\right.$ method of a transaction $\left.T_{i}\right)$ in a history $H$ is said to be isolated or atomic if for any other event $e_{p q r}\left(r^{t h}\right.$ event of method $\left.m_{p q}\right)$ belonging to some other method $m_{p q}$ of transaction $T_{p}$ either $e_{p q r}$ occurs before $\operatorname{inv}\left(m_{i j}\right)$ or after $\operatorname{rsp}\left(m_{i j}\right)$.

Sequential Histories: A history $H$ is said to be sequential (term used in [12, 13]) if all the methods in it are complete and isolated. From now onwards, most of our discussion would relate to sequential histories.

Since in sequential histories all the methods are isolated, we treat each method as a whole without referring to its $i n v$ and $r s p$ events. For a sequential history $H$, we construct the completion of $H$, denoted $\bar{H}$, by inserting $\operatorname{try} A_{k}(\mathscr{A})$ immediately after the last method of every transaction $T_{k} \in \operatorname{live}(H)$. Since all the methods in a sequential history are complete, this definition only has to take care of completed transactions.

Consider a sequential history $H$. Let $m_{i j}(h t, k, v / n i l)$ be the first method of $T_{i}$ in $H$ operating on the key $k$ as $H$.firstKeyMth $\left(\langle h t, k\rangle, T_{i}\right)$, where $m_{i j}$ stands for $j^{t h}$ method of $i^{t h}$ transaction. For a method $m_{i x}(h t, k, v)$ which is not the first method on $\langle h t, k\rangle$ of $T_{i}$ in $H$, we denote its previous method on $k$ of $T_{i}$ as $m_{i j}(h t, k, v)=$ H.prevKeyMth $\left(m_{i x}, T_{i}\right)$.

Real-time Order and Serial Histories: Given a history $H,<_{H}$ orders all the events

in $H$. For two complete methods $m_{i j}, m_{p q}$ in methods $(H)$, we denote $m_{i j} \prec_{H}^{M R} m_{p q}$ if $\operatorname{rsp}\left(m_{i j}\right)<_{H} \operatorname{inv}\left(m_{p q}\right)$. Here MR stands for method real-time order. It must be noted that all the methods of the same transaction are ordered. Similarly, for two transactions $T_{i}, T_{p}$ in $\operatorname{term}(H)$, we denote $\left(T_{i} \prec_{H}^{T R} T_{p}\right)$ if $\left(T_{i}\right.$.lastEvt $<_{H} T_{p}$. firstEvt $)$. Here TR stands for transactional real-time order. 
We define a history $H$ as serial [14] or t-sequential [13] if all the transactions in $H$ have terminated and can be totally ordered w.r.t $\prec_{T R}$, i.e. all the transactions execute one after the other without any interleaving. Intuitively, a history $H$ is serial if all its transactions can be isolated. Formally, $\left\langle(H\right.$ is serial $) \Longrightarrow\left(\forall T_{i} \in \operatorname{txns}(H):\left(T_{i} \in\right.\right.$ $\left.\operatorname{term}(H)) \wedge\left(\forall T_{i}, T_{p} \in \operatorname{txns}(H):\left(T_{i} \prec_{H}^{T R} T_{p}\right) \vee\left(T_{p} \prec_{H}^{T R} T_{i}\right)\right)\right\rangle$. Since all the methods within a transaction are ordered, a serial history is also sequential.

Valid Histories: A rv_method (t_delete and t_lookup) $r v m_{i j}$ on key $k$ is valid if it returns the value updated by any of the previously committed transaction that updated key $k$. A history $H$ is said to valid if all the rv_methods of $\mathrm{H}$ are valid.

Legal Histories: We define the legality of rv_methods on sequential histories which we use to define correctness criterion as opacity [8]. Consider a sequential history $H$ having a rv_method $r v m_{i j}(h t, k, v)($ with $v \neq n u l l)$ as $j^{t h}$ method belonging to transaction $T_{i}$. We define this rvm method to be legal if:

Rule 1 If the $r v m_{i j}$ is not the first method of $T_{i}$ to operate on $\langle h t, k\rangle$ and $m_{i x}$ is the previous method of $T_{i}$ on $\langle h t, k\rangle$. Formally, $r v m_{i j} \neq H$. firstKeyMth $\left(\langle h t, k\rangle, T_{i}\right)$ $\wedge\left(m_{i x}\left(h t, k, v^{\prime}\right)=\right.$ H.prevKeyMth $\left.\left(\langle h t, k\rangle, T_{i}\right)\right)$ (where $v^{\prime}$ could be null). Then,

(a) If $m_{i x}\left(h t, k, v^{\prime}\right)$ is a $t_{-} i n s e r t$ method then $v=v^{\prime}$.

(b) If $m_{i x}\left(h t, k, v^{\prime}\right)$ is a $t$ lookup method then $v=v^{\prime}$.

(c) If $m_{i x}\left(h t, k, v^{\prime}\right)$ is a $t_{-}$delete method then $v=$ null.

In this case, we denote $m_{i x}$ as the last update method of $r v m_{i j}$, i.e., $m_{i x}\left(h t, k, v^{\prime}\right)=H . l a s t U p d t\left(r v m_{i j}(h t, k, v)\right)$.

Rule 2 If $r v m_{i j}$ is the first method of $T_{i}$ to operate on $\langle h t, k\rangle$ and $v$ is not null. Formally, $r v m_{i j}(h t, k, v)=H$.firstKeyMth $\left(\langle h t, k\rangle, T_{i}\right) \wedge(v \neq n u l l)$. Then,

(a) There is a $t_{-}$insert method $t_{-}$insert pq $(h t, k, v)$ in methods $(H)$ such that $T_{p}$ committed before $r v m_{i j}$. Formally, $\left\langle\exists t\right.$ insert $_{p q}(h t, k, v) \in$ methods $(H)$ : $\left.\operatorname{try}_{p} \prec_{H}^{M R} r v m_{i j}\right\rangle$.

(b) There is no other update method $u p_{x y}$ of a transaction $T_{x}$ operating on $\langle h t, k\rangle$ in methods $(H)$ such that $T_{x}$ committed after $T_{p}$ but before $r v m_{i j}$. Formally, $\left\langle\nexists u p_{x y}\left(h t, k, v^{\prime \prime}\right) \in \operatorname{methods}(H): \operatorname{try} C_{p} \prec_{H}^{M R} \operatorname{try} C_{x} \prec_{H}^{M R}\right.$ $\left.r v m_{i j}\right\rangle$. 
In this case, we denote $\operatorname{try} C_{p}$ as the last update method of $r v m_{i j}$, i.e., $\operatorname{try} C_{p}(h t, k, v)=$ H.lastUpdt $\left(\operatorname{rvm}_{i j}(h t, k, v)\right)$.

Rule 3 If $r v m_{i j}$ is the first method of $T_{i}$ to operate on $\langle h t, k\rangle$ and $v$ is null. Formally, $r v m_{i j}(h t, k, v)=H . f i r s t K \operatorname{eyMth}\left(\langle h t, k\rangle, T_{i}\right) \wedge(v=n u l l)$. Then,

(a) There is $t$ delete method $t_{-}$delete $e_{p q}\left(h t, k, v^{\prime}\right)$ in $\operatorname{methods}(H)$ such that $T_{p}$ committed before $r v m_{i j}$. Formally, $\left\langle\exists t_{-}\right.$delete $p q$ $\left.\left(h t, k, v^{\prime}\right) \in \operatorname{methods}(H): \operatorname{try} C_{p} \prec_{H}^{M R} r v m_{i j}\right\rangle$. Here $v^{\prime}$ could be null.

(b) There is no other update method $u p_{x y}$ of a transaction $T_{x}$ operating on $\langle h t, k\rangle$ in methods $(H)$ such that $T_{x}$ committed after $T_{p}$ but before $r v m_{i j}$. Formally, $\left\langle\nexists u p_{x y}\left(h t, k, v^{\prime \prime}\right) \in \operatorname{methods}(H): \operatorname{try} C_{p} \prec_{H}^{M R} \operatorname{try} C_{x} \prec_{H}^{M R}\right.$ $\left.r v m_{i j}\right\rangle$.

In this case, we denote $\operatorname{try} C_{p}$ as the last update method of $r v m_{i j}$, i.e., $\operatorname{try} C_{p}(h t, k, v)$ $=H . l a s t U p d t\left(r v m_{i j}(h t, k, v)\right)$.

We assume that when a transaction $T_{i}$ operates on key $k$ of a hash-table $h t$, the result of this method is stored in $\operatorname{local}_{\log s}$ of $T_{i}, t x \log _{i}$ for later methods to reuse. Thus, only the first rv_method operating on $\langle h t, k\rangle$ of $T_{i}$ accesses the shared memory. The other rv_methods of $T_{i}$ operating on $\langle h t, k\rangle$ do not access the shared memory and they see the effect of the previous method from the $\operatorname{local} \log s, t x \log _{i}$. This idea is utilized in Rule 1. With reference to Rule 2 and Rule 3, it is possible that $T_{x}$ could have aborted before $r v m_{i j}$.

Coming to $t_{-i n s e r t}$ methods, since a $t_{-}$insert method always returns $o k$ as they overwrite the node if already present therefore they always take effect on the $h t$. Thus, we denote all $t$ insert methods as legal and only give legality definition for rv_method. We denote a sequential history $H$ as legal or linearized if all its rvm methods are legal. We formally prove the legality of the proposed OPT-MVOSTMs in Section 6

Opacity: It is a correctness-criteria for STMs [8]. A sequential history $H$ is said to be opaque if there exists a serial history $S$ such that: (1) $S$ is equivalent to $\bar{H}$, i.e., $\operatorname{evts}(\bar{H})=\operatorname{evts}(S)$ (2) $S$ is legal and (3) $S$ respects the transactional real-time order of $H$, i.e., $\prec_{H}^{T R} \subseteq \prec_{S}^{T R}$.

Finally, we show that history generated by OPT-MVOSTMs satisfy correctness 
criteria as opaque.

\section{Graph Characterization of Opacity}

To prove that an STM system satisfies opacity, it is useful to consider graph characterization of histories. In this section, we describe the graph characterization of Guerraoui and Kapalka [11] modified for sequential histories.

Consider a history $H$ which consists of multiple version for each t-object. The graph characterization uses the notion of version order. Given $H$ and a t-object $k$, we define a version order for $k$ as any (non-reflexive) total order on all the versions of $k$ ever created by committed transactions in $H$. It must be noted that the version order may or may not be the same as the actual order in which the versions of $k$ are generated in $H$. A version order of $H$, denoted as $\ll_{H}$ is the union of the version orders of all the t-objects in $H$.

Consider the history $H 3$ as shown in Figure $3: l u_{1}\left(k_{x, 0}, n u l l\right), l u_{2}\left(k_{x, 0}, n u l l\right), l u_{1}$ $\left(k_{y, 0}, n u l l\right), l u_{3}\left(k_{z, 0}, n u l l\right)$, ins $_{1}\left(k_{x, 1}, v_{11}\right)$, ins $_{3}\left(k_{y, 3}, v_{31}\right)$, ins $_{2}\left(k_{y, 2}, v_{21}\right)$, ins $_{1}\left(k_{z, 1}\right.$, $\left.v_{12}\right), c_{1}, c_{2}, l u_{4}\left(k_{x, 1}, v_{11}\right), l u_{4}\left(k_{y, 2}, v_{21}\right), \operatorname{ins}_{3}\left(k_{z, 3}, v_{32}\right), c_{3}, l u_{4}\left(k_{z, 1}, v_{12}\right), l u_{5}\left(k_{x, 1}\right.$, $\left.v_{11}\right), l u_{6}\left(k_{y, 2}, v_{21}\right), c_{4}, c_{5}, c_{6}$. Using the notation that a committed transaction $T_{i}$ writing to $k_{x}$ creates a version $k_{x, i}$, a possible version order for $H 3 \ll_{H 3}$ is: $\left\langle k_{x, 0} \ll\right.$ $\left.k_{x, 1}\right\rangle,\left\langle k_{y, 0} \ll k_{y, 2} \ll k_{y, 3}\right\rangle,\left\langle k_{z, 0} \ll k_{z, 1} \ll k_{z, 3}\right\rangle$.

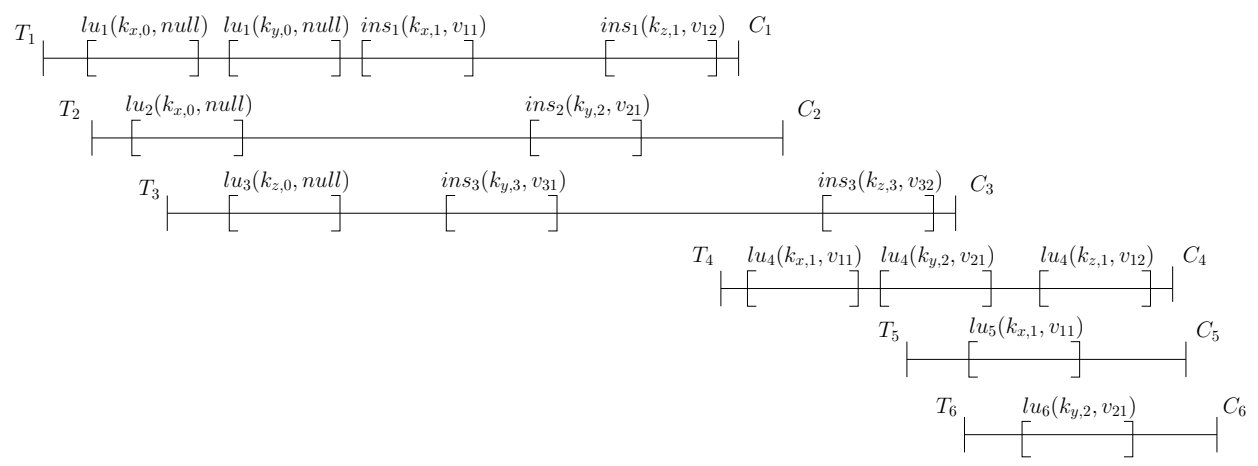

Figure 3: History $H 3$ in time line view

We define the graph characterization based on a given version order. Consider a history $H$ and a version order $\ll$. We then define a graph (called opacity graph) on $H$ using $\ll$, denoted as $O P G(H, \ll)=(V, E)$. The vertex set $V$ consists of a vertex for 
each transaction $T_{i}$ in $\bar{H}$. The edges of the graph are of three kinds and are defined as follows:

1. $r t$ (real-time) edges: If the commit of $T_{i}$ happens before beginning of $T_{j}$ in $H$, then there exist a real-time edge from $v_{i}$ to $v_{j}$. We denote set of such edges as $r t(H)$.

2. $r v f$ (return value-from) edges: If $T_{j}$ invokes rv method on key $k_{1}$ from $T_{i}$ which has already been committed in $H$, then there exists a return value-from edge from $v_{i}$ to $v_{j}$. If $T_{i}$ is having upd_method as insert on the same key $k_{1}$ then ins $_{i}\left(k_{1, i}, v_{i 1}\right)<_{H} c_{i}<_{H} r v m_{j}\left(k_{1, i}, v_{i 1}\right)$. If $T_{i}$ is having upd_method as delete on the same key $k_{1}$ then $\operatorname{del}_{i}\left(k_{1, i}\right.$, null $)<_{H} c_{i}<_{H} \operatorname{rvm}_{j}\left(k_{1, i}\right.$, null $)$. We denote set of such edges as $r v f(H)$.

3. $m v$ (multi-version) edges: This is based on version order. Consider a triplet with successful methods as $u p_{i}\left(k_{1, i}, u\right), r v m_{j}\left(k_{1, i}, u\right), u p_{k}\left(k_{1, k}, v\right)$, where $u \neq v$. As we can observe it from $r v m_{j}\left(k_{1, i}, u\right), c_{i}<_{H} r v m_{j}\left(k_{1, i}, u\right)$. if $k_{1, i} \ll k_{1, k}$ then there exist a multi-version edge from $v_{j}$ to $v_{k}$. Otherwise $\left(k_{1, k} \ll k_{1, i}\right)$, there exist a multi-version edge from $v_{k}$ to $v_{i}$. We denote set of such edges as $m v(H, \ll)$.

We now show that if a version order $\ll$ exists for a history $H$ such that it is acyclic, then $H$ is opaque.

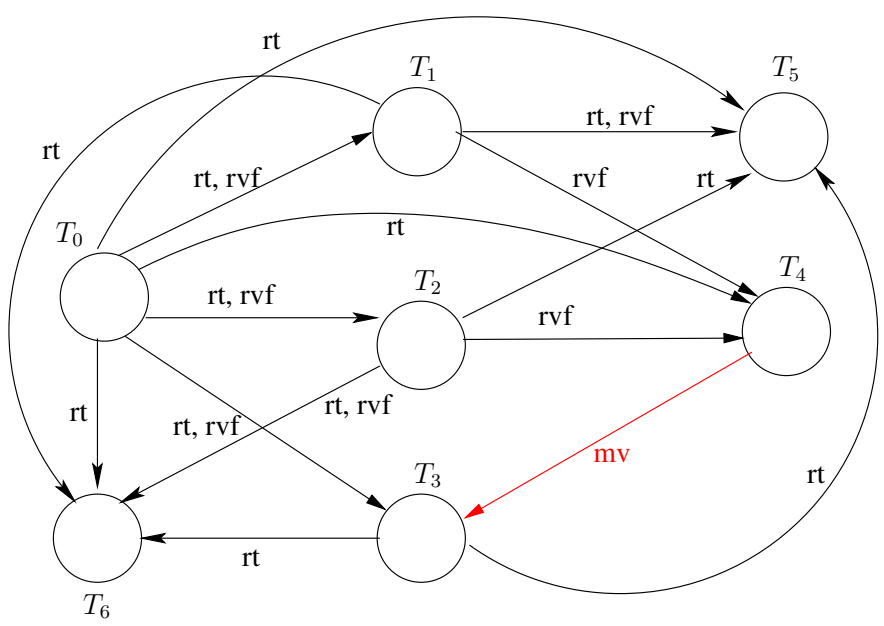

Figure 4: $O P G\left(H 3, \ll_{H 3}\right)$ 
Using this construction, the $O P G\left(H 3, \ll_{H 3}\right)$ for history $H 3$ and $\ll_{H 3}$ is given above is shown in Figure 4 The edges are annotated. The only mv edge from $T_{4}$ to $T_{3}$ is because of t-objects $k_{y}, k_{z} . T_{4}$ lookups value $v_{12}$ for $k_{z}$ from $T_{1}$ whereas $T_{3}$ also inserts $v_{32}$ to $k_{z}$ and commits before $l u_{4}\left(k_{z, 1}, v_{12}\right)$.

Given a history $H$ and a version order $\ll$, consider the graph $O P G(\bar{H}, \ll)$. While considering the $r t$ edges in this graph, we only consider the real-time relation of $H$ and not $\bar{H}$. It can be seen that $\prec_{H}^{R T} \subseteq \prec \frac{R T}{H}$ but with this assumption, $r t(H)=r t(\bar{H})$. Hence, we get the following property,

Property 1. The graphs $O P G(H, \ll)$ and $O P G(\bar{H}, \ll)$ are the same for any history $H$ and $\ll$.

Definition 1. For a t-sequential history $S$, we define a version order $\ll_{S}$ as follows: For two version $k_{x, i}, k_{x, j}$ created by committed transactions $T_{i}, T_{j}$ in $S,\left\langle k_{x, i} \ll_{S}\right.$ $\left.k_{x, j} \Leftrightarrow T_{i}<_{S} T_{j}\right\rangle$.

Now we show the correctness of our graph characterization using the following lemmas and theorem.

Lemma 2. Consider a legal t-sequential history $S$. Then the graph $O P G\left(S, \ll_{S},\right)$ is acyclic.

Proof: We numerically order all the transactions in $S$ by their real-time order by using a function $\operatorname{ord}$. For two transactions $T_{i}, T_{j}$, we define $\operatorname{ord}\left(T_{i}\right)<\operatorname{ord}\left(T_{j}\right) \Leftrightarrow T_{i}<_{S} T_{j}$. Let us analyze the edges of $O P G\left(S, \ll_{S},\right)$ one by one:

- rt edges: It can be seen that all the rt edges go from a lower ord transaction to a higher ord transaction.

- rvf edges: If $T_{j}$ lookups $k_{x}$ from $T_{i}$ in $S$ then $T_{i}$ is a committed transaction with $\operatorname{ord}\left(T_{i}\right)<\operatorname{ord}\left(T_{j}\right)$. Thus, all the rvf edges from a lower ord transaction to a higher ord transaction.

- mv edges: Consider a successful rv_method $r v m_{j}\left(k_{x}, u\right)$ and a committed transaction $T_{k}$ writing $v$ to $k_{x}$ where $u \neq v$. Let $c_{i}$ be $r v m_{j}\left(k_{x}, u\right)$ 's lastWrite. Thus, 
$u_{i}\left(k_{x, i}, u\right) \in \operatorname{evts}\left(T_{i}\right)$. Thus, we have that $\operatorname{ord}\left(T_{i}\right)<\operatorname{ord}\left(T_{j}\right)$. Now there are two cases w.r.t $T_{i}$ : (1) Suppose $\operatorname{ord}\left(T_{k}\right)<\operatorname{ord}\left(T_{i}\right)$. We now have that $T_{k} \ll T_{i}$. In this case, the mv edge is from $T_{k}$ to $T_{i}$. (2) Suppose $\operatorname{ord}\left(T_{i}\right)<\operatorname{ord}\left(T_{k}\right)$ which implies that $T_{i} \ll T_{k}$. Since $S$ is legal, we get that $\operatorname{ord}\left(T_{j}\right)<\operatorname{ord}\left(T_{k}\right)$. This case also implies that there is an edge from $\operatorname{ord}\left(T_{j}\right)$ to $\operatorname{ord}\left(T_{k}\right)$. Hence, in this case as well the mv edges go from a transaction with lower ord to a transaction with higher ord.

Thus, in all the three cases the edges go from a lower ord transaction to higher ord transaction. This implies that the graph is acyclic.

Lemma 3. Consider two histories $H, H^{\prime}$ that are equivalent to each other. Consider a version order $\ll_{H}$ on the t-objects created by $H$. The mv edges $m v\left(H, \ll_{H}\right)$ induced by $\ll_{H}$ are the same in $H$ and $H^{\prime}$.

Proof: Since the histories are equivalent to each other, the version order $\ll_{H}$ is applicable to both of them. It can be seen that the mv edges depend only on events of the history and version order $\ll$. It does not depend on the ordering of the events in $H$. Hence, the mv edges of $H$ and $H^{\prime}$ are equivalent to each other.

Using these lemmas, we prove the following theorem.

Theorem 4. A valid history $H$ is opaque iff there exists a version order $\ll_{H}$ such that $O P G\left(H, \ll_{H}\right)$ is acyclic.

Proof: (if part): Here we have a version order $\ll_{H}$ such that $G_{H}=O P G(H, \ll)$ is acyclic. Now we have to show that $H$ is opaque. Since the $G_{H}$ is acyclic, a topological sort can be obtained on all the vertices of $G_{H}$. Using the topological sort, we can generate a t-sequential history $S$. It can be seen that $S$ is equivalent to $\bar{H}$. Since $S$ is obtained by a topological sort on $G_{H}$ which maintains the real-time edges of $H$, it can be seen that $S$ respects the rt order of $H$, i.e $\prec_{H}^{R T} \subseteq \prec_{S}^{R T}$.

Similarly, since $G_{H}$ maintains return value-from (rvf) order of $H$, it can be seen that if $T_{j}$ lookups $k_{x}$ from $T_{i}$ in $H$ then $T_{i}$ terminates before $l u_{j}\left(k_{x}\right)$ and $T_{j}$ in $S$. Thus, $S$ is valid. Now it remains to be shown that $S$ is legal. We prove this using 
contradiction. Assume that $S$ is not legal. Thus, there is a successful rv_method $r v m_{j}\left(k_{x}, u\right)$ such that its lastWrite in $S$ is $c_{k}$ and $T_{k}$ updates value $v(\neq u)$ to $k_{x}$, i.e $u p_{k}\left(k_{x, k}, v\right) \in \operatorname{evts}\left(T_{k}\right)$. Further, we also have that there is a transaction $T_{i}$ that inserts $u$ to $k_{x}$, i.e $u p_{i}\left(k_{x, i}, u\right) \in \operatorname{evts}\left(T_{i}\right)$. Since $S$ is valid, as shown above, we have that $T_{i} \prec S T T_{k} \prec_{S}^{R T} T_{j}$.

Now in $\ll_{H}$, if $k_{x, k} \ll_{H} k_{x, i}$ then there is an edge from $T_{k}$ to $T_{i}$ in $G_{H}$. Otherwise $\left(k_{x, i} \ll_{H} k_{x, k}\right)$, there is an edge from $T_{j}$ to $T_{k}$. Thus, in either case, $T_{k}$ can not be in between $T_{i}$ and $T_{j}$ in $S$ contradicting our assumption. This shows that $S$ is legal.

(Only if part): Here we are given that $H$ is opaque and we have to show that there exists a version order $\ll$ such that $G_{H}=O P G(H, \ll)(=O P G(\bar{H}, \ll)$, Property 1 ) is acyclic. Since $H$ is opaque there exists a legal t-sequential history $S$ equivalent to $\bar{H}$ such that it respects real-time order of $H$. Now, we define a version order for $S, \ll_{S}$ as in Definition 1. Since the $S$ is equivalent to $\bar{H}, \ll_{S}$ is applicable to $\bar{H}$ as well. From Lemma 2, we get that $G_{S}=\operatorname{OPG}\left(S, \ll_{S}\right)$ is acyclic. Now consider $G_{H}=\operatorname{OPG}\left(\bar{H}, \ll_{S}\right)$. The vertices of $G_{H}$ are the same as $G_{S}$. Coming to the edges,

- rt edges: We have that $S$ respects real-time order of $H$, i.e $\prec_{H}^{R T} \subseteq \prec_{S}^{R T}$. Hence, all the rt edges of $H$ are a subset of $S$.

- rvf edges: Since $\bar{H}$ and $S$ are equivalent, the return value-from relation of $\bar{H}$ and $S$ are the same. Hence, the rvf edges are the same in $G_{H}$ and $G_{S}$.

- mv edges: Since the version-order and the operations of the $H$ and $S$ are the same, from Lemma 3 it can be seen that $\bar{H}$ and $S$ have the same mv edges as well.

Thus, the graph $G_{H}$ is a subgraph of $G_{S}$. Since we already know that $G_{S}$ is acyclic from Lemma 2, we get that $G_{H}$ is also acyclic.

\section{OPT-MVOSTMs Design and Data Structure}

This section describes the design and data structure of optimized MVOSTMs (or OPT-MVOSTMs). Here, we propose hash-table and list based OPT-MVOSTMs as OPTHT-MVOSTM and OPT-list-MVOSTM respectively. OPT-MVOSTMs are generic for 

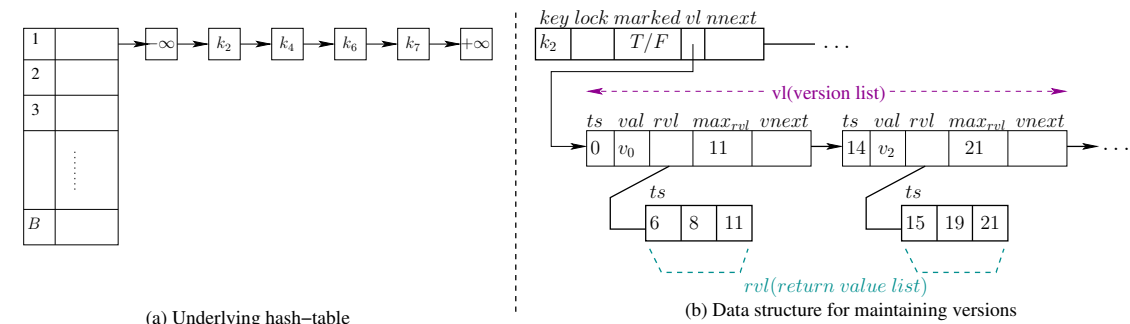

Figure 5: Optimized HT-MVOSTM design

other data structure as well. OPT-HT-MVOSTM is a hash-table based OPT-MVOSTM that explores the idea of multiple versions in OSTMs for hash-table object to achieve greater concurrency. The design of OPT-HT-MVOSTM is similar to HT-MVOSTM [1] consisting of $B$ buckets. All the keys of the hash-table in the range $\mathscr{K}$ are statically allocated to one of these buckets.

Each bucket consists of linked-list of nodes along with two sentinel nodes head and tail with values $-\infty$ and $+\infty$ respectively. The structure of each node is as $\langle k e y$, lock, marked, $v l$, nnext $\rangle$. The key is a unique value from the set of all keys $\mathscr{K}$. All the nodes are stored in increasing order in each bucket as shown in Figure 5(a), similar to any linked-list based concurrent set implementation [7, 15]. In the rest of the document, we use the terms key and node interchangeably. To perform any operation on a key, the corresponding lock is acquired. marked is a boolean field which represents whether the key is deleted or not. The deletion is performed in a lazy manner similar to the concurrent linked-lists structure [7]. If the marked field is true then key corresponding to the node has been logically deleted; otherwise, it is present. The $v l$ field of the node points to the version list (shown in Figure 5(b)) which stores multiple versions corresponding to the key. The last field of the node is nnext which stores the address of the next node. It can be seen that the list of keys in a bucket is as an extension of lazy-list [7]. Given a node $n$ in the linked-list of bucket $B$ with key $k$, we denote its fields as n.key (or k.key), n.lock (or k.lock), n.marked (or k.marked), n.vl (or k.vl), n.nnext (or k.nnext).

The structure of each version in the $v l$ of a key $k$ is $\left\langle t s, v a l, r v l, \max _{r v l}\right.$, vnext $\rangle$ as shown in Figure 5(b). The field $t s$ denotes the unique timestamp of the version. In 
our algorithm, every transaction is assigned a unique timestamp when it begins which is also its $i d$. Thus $t s$ of this version is the timestamp of the transaction that created it. All the versions in the $v l$ of $k$ are sorted by $t s$. Since the timestamps are unique, we denote a version, ver of a node $n$ with key $k$ having $t s j$ as $n . v l[j] . v e r$ or $k . v l[j] . v e r$. The corresponding fields in the version as $k . v l[j] . t s, k . v l[j] . v a l, k . v l[j] . r v l, k . v l[j] . \max _{r v l}$, $k . v l[j] . v n e x t$.

The field val contains the value updated by an update transaction. If this version is created by an insert method $t_{-}$insert $t_{i}(h t, k, v)$ by transaction $T_{i}$, then val will be $v$. On the other hand, if the method is $t_{-}$delete $_{i}(h t, k, v)$ then $v a l$ will be $n u l l$. In this case, as per the algorithm, the node of key $k$ will also be marked. OPT-HT-MVOSTM algorithm does not immediately physically remove deleted keys from the hash-table. The need for this is explained below. Thus an rv_method (t_delete or $t_{-}$lookup) on key $k$ can return null when it does not find the key or encounters a $n u l l$ value for $k$.

The $r v l$ field stands for return value list which is a list of all the transactions that executed rv_method on this version, i.e., those transactions which returned val. The first optimization in OPT-HT-MVOSTM to reduce the traversal time of $r v l$, we have used $\max _{r v l}$ which contains the maximum $t s$ of the transaction that executed rv_method on this version. The field vnext points to the next available version of that key.

In order to increase the efficiency and utilize the memory properly, We propose two variants of OPT-HT-MVOSTM as follows: First, we apply garbage collection (or GC) on the versions and propose OPT-HT-MVOSTM-GC. It maintains unbounded versions in $v l$ (the length of the list) while deleting the unwanted versions using garbage collection scheme. Second, we propose OPT-HT-KOSTM which maintains the bounded number of versions such as $K$ and improves the efficiency further. Whenever a new version $v e r$ is created and is about to be added to $v l$, the length of $v l$ is checked. If the length becomes greater than $K$, the version with lowest $t s$ (i.e., the oldest) is replaced with the new version ver and thus maintaining the length back to $K$.

We propose OPT-list-MVOSTMs while considering the bucket size as 1 in OPT-HTMVOSTM. Along with this, we propose two variants of OPT-list-MVOSTM as OPT-listMVOSTM-GC and OPT-list-KOSTM which applies the garbage collection scheme in unbounded versions and bounded $K$ versions for list based object respectively similar 


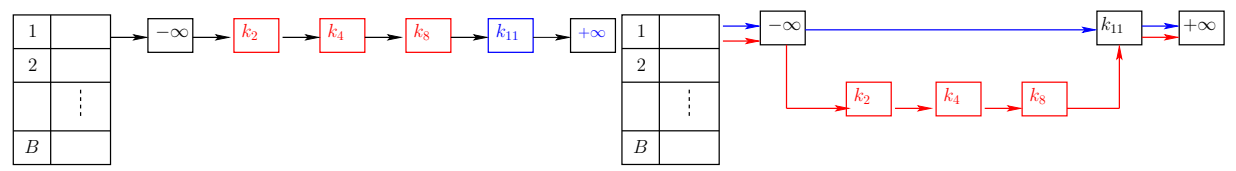

Figure 6: Searching $k_{11}$ over lazy-list

Figure 7: Searching $k_{11}$ over lazyrb-list

to OPT-HT-MVOSTM.

Marked Version Nodes: OPT-HT-MVOSTM stores keys even after they have been deleted (the version of the nodes which have marked field as true). This is because some other concurrent transactions could read from a different version of this key and not the null value inserted by the deleting transaction. Consider for instance the transaction $T_{1}$ performing $l u_{1}\left(h t, k_{2}, v_{0}\right)$ as shown in Figure 2 (b). Due to the presence of previous version $v_{0}$, OPT-HT-MVOSTM returns this earlier version $v_{0}$ for $l u_{1}\left(h t, k_{2}, v_{0}\right)$ method. Whereas, it is not possible for HT-OSTM to return the version $v_{0}$ because $k_{1}$ has been removed from the system by delete method of higher timestamp transaction $T_{2}$ than $T_{1}$. In that case, $T_{1}$ would have to be aborted. Thus as explained in Section 11, storing multiple versions increases the concurrency.

To store deleted keys along with the live keys (or unmarked node) in a lazy-list will increase the traversal time to access unmarked nodes. Consider Figure 6, in which there are four keys $\left\langle k_{2}, k_{4}, k_{8}, k_{11}\right\rangle$ present in the list. Here $\left\langle k_{2}, k_{4}, k_{8}\right\rangle$ are marked (or deleted) nodes while $k_{11}$ is unmarked. Now, consider accessing the key $k_{11}$ by OPT-HT-MVOSTM as a part of one of its methods. Then OPT-HT-MVOSTM would have to unnecessarily traverse the marked nodes to reach key $k_{11}$.

This motivated us to modify the lazy-list structure of nodes in each bucket to form a skip list based on red and blue links. We denote it as red-blue lazy-list or lazyrb-list. This idea was earlier explored by Peri et al. in developing OSTMs [6]. lazyrb-list consists of nodes with two links, red link (or RL) and blue link (or BL). The node which is not marked (or not deleted) are accessible from the head via BL. While all the nodes including the marked ones can be accessed from the head via RL. With this modification, let us consider the above example of accessing unmarked key $k_{11}$. It can be seen that $k_{11}$ can be accessed much more quickly through $\mathrm{BL}$ as shown in Figure 7 Using the idea of lazyrb-list, we have modified the structure of each node as $\langle$ key, lock, marked, 
$v l, R L, B L\rangle$. Further, for a bucket $B$, we denote its linked-list as B.lazyrb-list.

\section{Working of OPT-HT-MVOSTM}

OPT-HT-MVOSTM exports $t$ _begin, t_insert, t_delete, t_lookup, and tryC methods

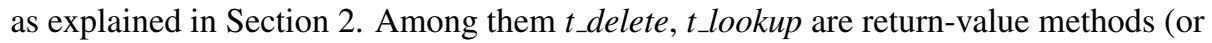
rv_methods) while $t_{-}$insert, $t \_d e l e t e$ are update methods (or upd_methods). We treat t_delete as both rv_method as well as upd_method. The rv_methods return the current value of the key. The upd_methods, update to the keys are first noted down in the local $\log , t x \log$. Then in the tryC method after successful validations of these updates are transferred to the shared memory. We now explain the working of each method as follows:

tbbegin(): A thread invokes a new transaction $T_{i}$ using this method. The transaction $T_{i}$ local $\log t x \log _{i}$ is initialized at Line 2 . This method returns a unique id to the invoking thread by incrementing an atomic counter at Line 3 This unique id is also the timestamp of the transaction $T_{i}$. For convenience, we use the notation that $i$ is the timestamp (or id) of the transaction $T_{i}$.

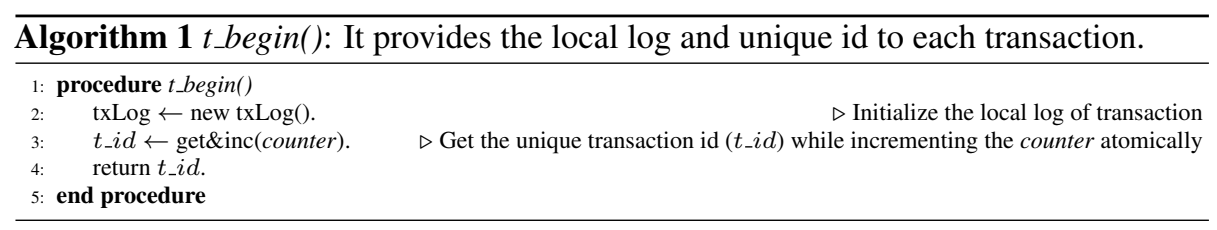

rv_methods: It can be either $t_{-}$delete $(h t, k, v)$ or $t_{-} \operatorname{lookup}(h t, k, v)$. Both these methods return the current value of key $k$. Algorithm 2 gives the high level overview of these methods. First, the algorithm checks to see if the given key is already in the local log, $t x \log _{i}$ of $T_{i}$ (Line 7). If the key is already there then the current rv method is not the first method on $k$ and is a subsequent method of $T_{i}$ on $k$. So, we can return the value of $k$ from the $t x \log _{i}$.

If the key is not present in the $t x \log _{i}$, then OPT-HT-MVOSTM searches into shared memory. Specifically, it searches the bucket to which $k$ belongs to. Every key in the range $\mathscr{K}$ is statically allocated to one of the $B$ buckets. So the algorithms search 
for $k$ in the corresponding bucket, say $B_{k}$ to identify the appropriate location, i.e., identify the correct predecessor or pred and current or curr keys in the lazyrb-list of $B_{k}$ without acquiring any locks similar to the search in lazy-list [7]. Since each key has two links, RL and BL, the algorithm identifies four node references: two pred and two curr according to red and blue links. They are stored in the form of an array with preds[0] and currs[1] corresponding to blue links; preds[1] and currs $[0]$ corresponding to red links. If both preds[1] and currs $[0]$ nodes are unmarked then the pred,curr nodes of both red and blue links will be the same, i.e., preds $[0]=$ preds $[1]$ and currs $[0]=$ currs $[1]$. Thus depending on the marking of pred,curr nodes, a total of two, three or four different nodes will be identified. Here, the search ensures that preds[0].key $\leq$ preds[1].key $<k \leq$ currs[0].key $\leq$ currs[1].key.

Next, the re-entrant locks on all the pred,curr keys are acquired in increasing order to avoid the deadlock. Then all the pred and curr keys are validated by $r v_{-}-$ Validation() in Line 12 as follows: (1) If pred and curr nodes of blue links are not marked, i.e, ( $\neg$ preds[0].marked) \&\& ( $\neg$ currs[1].marked). (2) If the next links of both blue and red pred nodes point to the correct curr nodes: (preds $[0] \cdot B L=$ currs[1]) \&\& (preds $[1] . R L=\operatorname{currs}[0])$ at Line 74

If any of these checks fail, then the algorithm retries to find the correct pred and curr keys. It can be seen that the validation check is similar to the validation in concurrent lazy-list [7].

Next, we check if $k$ is in $B_{k}$.lazyrb-list. If $k$ is not in $B_{k}$, then we create a new node $n$ for $k$ as: $\langle k e y=k$, lock $=$ false, marked $=$ true, $v l=v e r, n n e x t=\phi\rangle$ and insert it into $B_{k}$.lazyrb-list such that it is accessible only via RL. This node will have a single version ver as $\left\langle t s=0, v a l=n u l l, r v l=i, \max _{r v l}=i, v n e x t=\phi\right\rangle$. Here invoking transaction $T_{i}$ is creating a version with timestamp 0 to ensure that rv_methods of other transactions will never abort. As we have explained in Figure 2 (b) of Section 1 . even after $T_{2}$ deletes $k_{2}$, the previous value of $v_{0}$ is still retained. Thus, when $T_{1}$ invokes $l u$ on $k_{2}$ after the delete on $k_{2}$ by $T_{2}, O P T-H T-M V O S T M$ will return $v_{0}$ (as previous value). Hence, each rv_method will find a version to read while maintaining the infinite version corresponding to each key $k$. marked field sets to true because it access by $R L$ only. In $r v l$ and $\max _{r v l}, T_{i}$ adds the timestamp as $i$ in it and vnext is initialized to 
empty value. Since $v a l$ is null and the $n$, this version and the node are not technically inserted into $B_{k}$.lazyrb-list.

If $k$ is in $B_{k}$.lazyrb-list then, $k$ is the same as currs [0] or currs[1] or both. Let $n$ be the node of $k$ in $B_{k}$.lazyrb-list. We then find the version of $n, v e r_{j}$ which has the timestamp $j$ such that $j$ has the largest timestamp smaller than $i$ (timestamp of $T_{i}$ ). Add $i$ to $v e r_{j}$ 's $r v l$ (Line 24p. $\max _{r v l}$ maintains the maximum timestamp among all rv_methods read from this version at Line 26 Then release the locks, update the local $\log t x \log _{i}$ in Line 29 and return the value stored in $v e r_{j} . v a l$ in Line 31

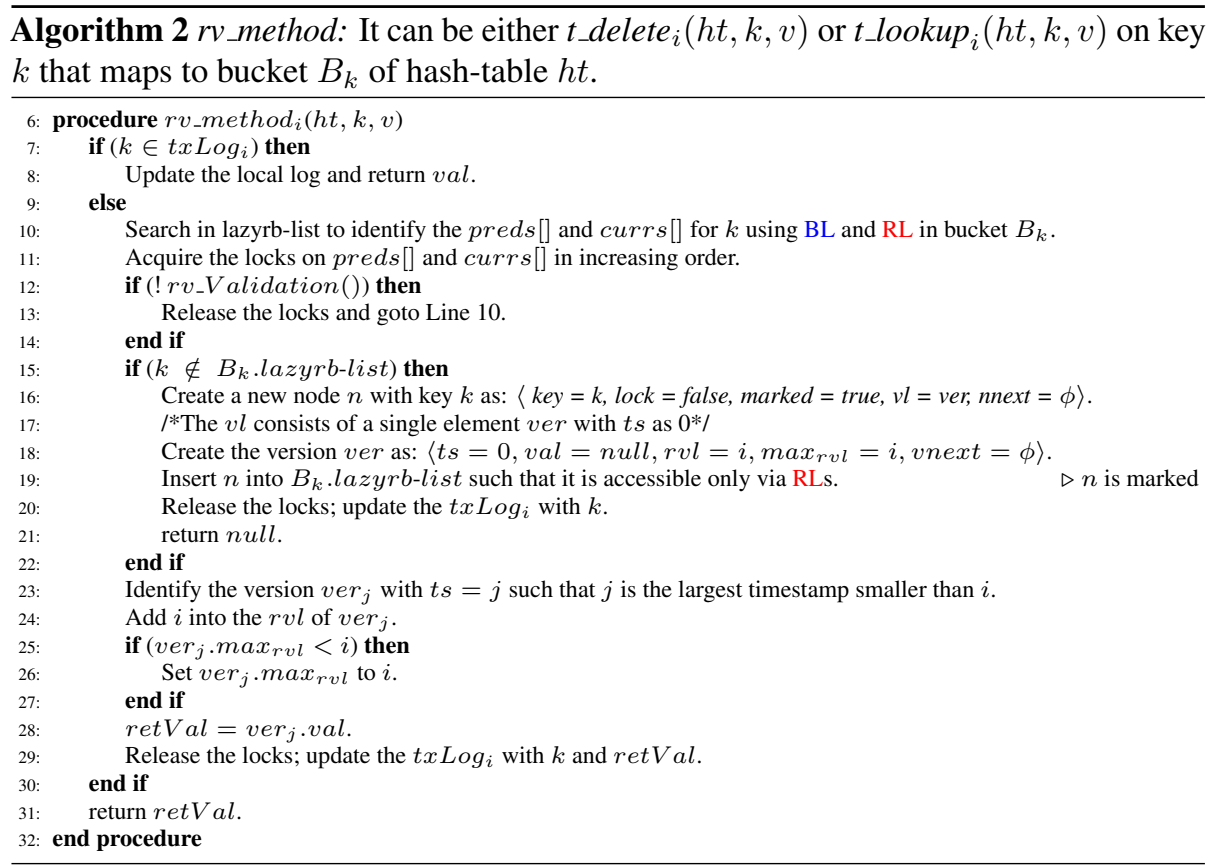

t insert(): This is another optimization done in OPT-HT-MVOSTMs to identify the early abort which prevents the work done by aborted transactions and saves time. The actual effect of the $t_{-}$insert() comes after the successful tryC method. First, t_insert() searches the key $k$ in the local $\log , t x \log _{i}$ of $T_{i}$ at Line 34 . If $k$ does not exist in the $t x \log _{i}$ then it identifies the appropriate location (pred and curr) of key $k$ using BL and RL (Line 35) in the lazyrb-list of $B_{k}$ without acquiring any locks similar to rv_method explained above. 


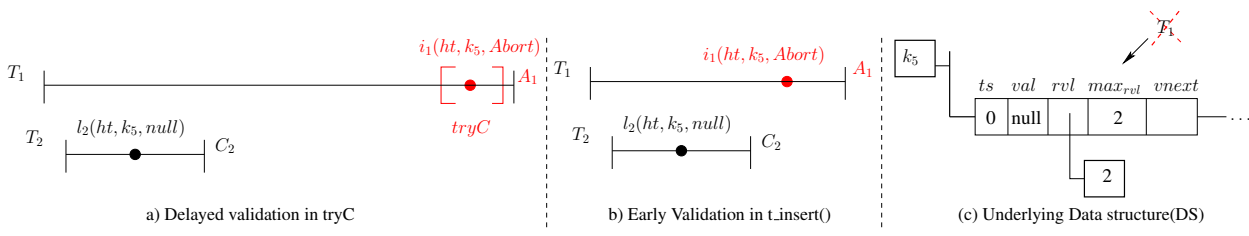

Figure 8: Advantage of early validation in t_insert()

Next, it acquires the re-entrant locks on all the pred and curr keys in increasing order. After that, all the pred and curr keys are validated by tryC_Validation in Line 37 as follows: (1) It does the $r v_{-}$Validation() as explained above in the rv_method. (2) If key $k$ exists in the $B_{k}$.lazyrb-list and let $n$ as a node of $k$. Then algorithm identifies the version of $n$, ver $_{j}$ which has the timestamp $j$ such that $j$ has the largest timestamp smaller than $i$ (timestamp of $T_{i}$ ) at Line 85. If $\max _{r v l}$ of $v e r_{j}$ is greater than timestamp $i$ at Line 86 then it returns Abort in Line 38 .

tryC_Validation() in t_insert() identifies the early abort of invalid transaction. The advantage of doing the early validation to save the significant computation of long running transaction which will abort in the future. Consider Figure 8 where two transaction $T_{1}$ and $T_{2}$ working on key $k_{5}$. In Figure 8 (a), $T_{1}$ aborts in tryC (delayed validation) because higher timestamp $T_{2}$ committed. But in Figure 8 (b), $T_{1}$ validates the t_insert() instantly by looking into the $\max _{r v l}$ of $k_{5}$ as shown in Figure 8 (c) and save its computation and returns abort.

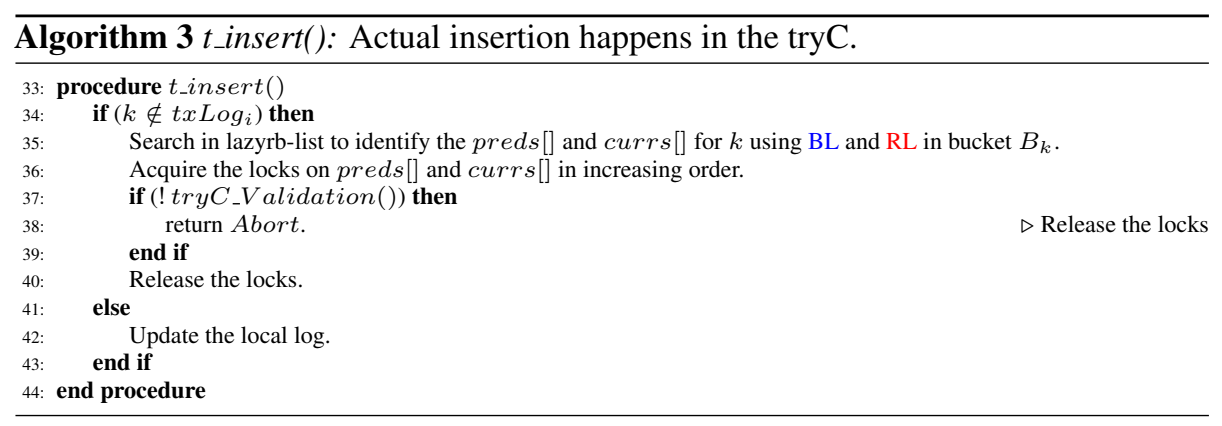

upd_methods: It can be either $t_{-}$insert $(h t, k, v)$ or $t_{-}$delete $(h t, k, v)$. Both the methods create a version corresponding to the key $k$. The actual effect of $t_{-}$insert and $t$ delete in shared memory will take place in tryC. Algorithm 4 represents the high level overview 
of $\operatorname{try} C$.

Initially, to avoid deadlocks, the algorithm sorts all the keys in increasing order which are present in the local $\log , t x \log _{i}$. In $\operatorname{try} C, t x \log _{i}$ consists of upd_methods (t_insert or $t_{-}$delete) only. For all the upd_methods $\left(o p n_{i}\right)$ it searches the key $k$ in the shared memory corresponding to the bucket $B_{k}$. It identifies the appropriate location (pred and curr) of key $k$ using BL and RL (Line 50) in the lazyrb-list of $B_{k}$ without acquiring any locks similar to rv_method explained above.

Next, it acquires the re-entrant locks on all the pred and curr keys in increasing order. After that, all the pred and curr keys are validated by tryC_Validation in Line 52 as explained in $t_{\text {_insert(). }}$.

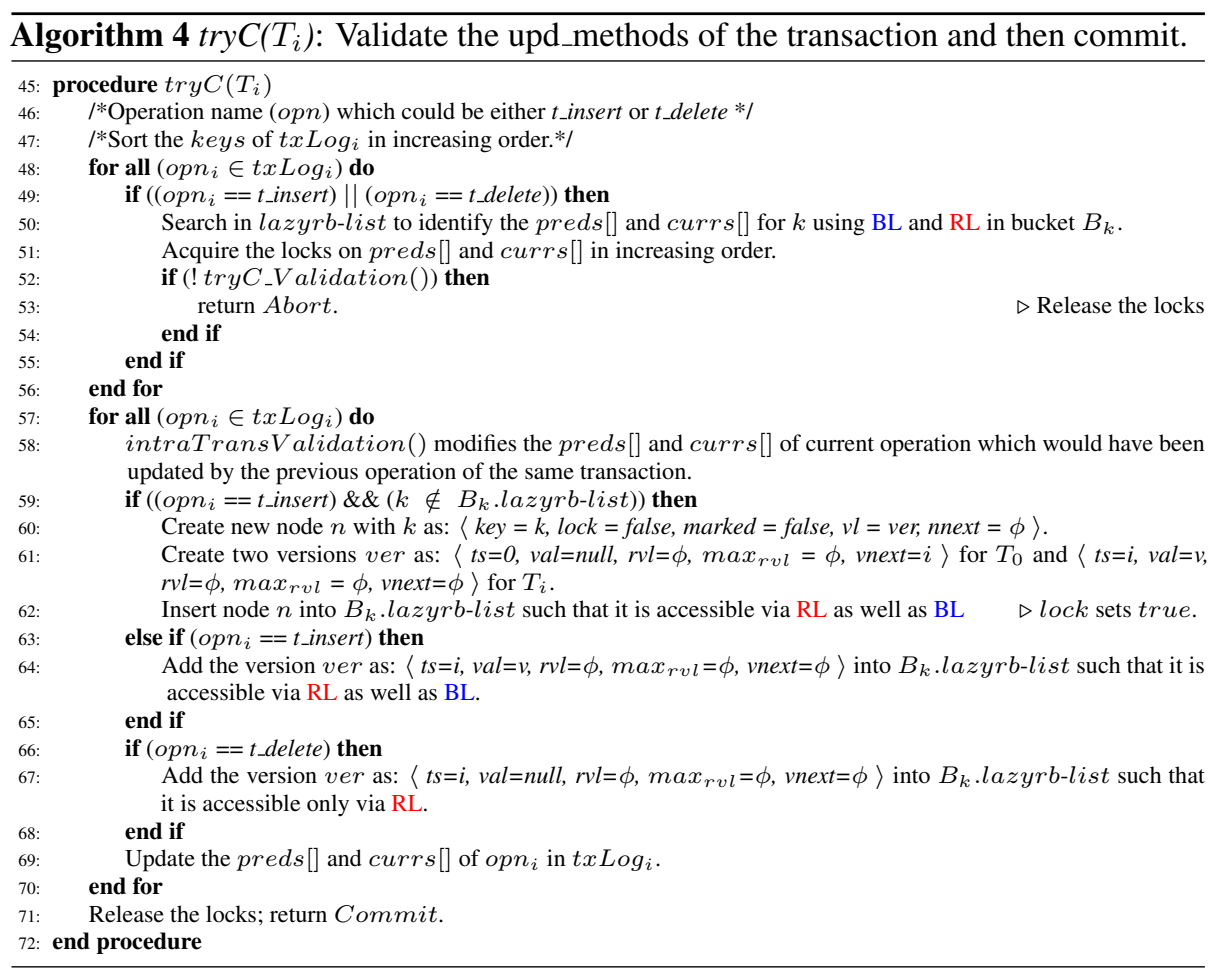

If tryC_Validation is successful then each upd_methods exist in $t x \log _{i}$ will take the effect in the shared memory after doing the intraTransValidation() in Line 58 If two upd_methods of the same transaction have at least one common shared node among its recorded pred and curr keys, then the previous upd_method effect may 
overwrite if the current upd_method of pred and curr keys are not updated according to the updates are done by the previous upd_method. Thus to solve this we have intraTransValidation() that modifies the pred and curr keys of current operation based on the previous operation in Line 58 .

Next, we check if upd_method is $t_{-}$insert and $k$ is in $B_{k}$.lazyrb-list. If $k$ is not in $B_{k}$, then create a new node $n$ for $k$ as $\langle k e y=k$, lock $=$ false, marked $=$ false, $v l=$ ver, nnext $=\phi\rangle$. This node will have two versions ver as $\langle t s=0$, val $=n u l l, r v l=$ $\phi, \max _{r v l}=\phi$, vnext $\left.=i\right\rangle$ for $T_{0}$ and $\left\langle t s=i, v a l=v, r v l=\phi, \max _{r v l}=\right.$ $\phi, v n e x t=\phi\rangle$ for $T_{i} . T_{i}$ is creating a version with timestamp 0 to ensure that rv_methods of other transactions will never abort. For second version, $i$ is the timestamp of the transaction $T_{i}$ invoking this method; marked field sets to false because the node is inserted in the BL. rvl, $\max _{r v l}$, and vnext are initialized to empty values. We set the $v a l$ as $v$ and insert $n$ into $B_{k}$.lazyrb-list such that it is accessible via RL as well as $\mathrm{BL}$ and set the lock field to be true (Line 62). If $k$ is in $B_{k}$.lazyrb-list then, $k$ is the same as currs [0] or currs [1] or both. Let $n$ be the node of $k$ in $B_{k}$.lazyrb-list. Then, we create the version ver as: $\left\langle t s=i, v a l=v, r v l=\phi, \max _{r v l}=\phi\right.$, vnext $\left.=\phi\right\rangle$ and insert the version into $B_{k}$.lazyrb-list such that it is accessible via RL as well as BL (Line 64).

Subsequently, we check if upd_method is $t_{-}$delete and $k$ is in $B_{k}$.lazyrb-list. Let $n$ be the node of $k$ in $B_{k}$.lazyrb-list. Then create the version ver as $\langle t s=i, v a l=$ $n u l l, r v l=\phi, \max _{r v l}=\phi$, vnext $\left.=\phi\right\rangle$ and insert the version into $B_{k}$.lazyrb-list such that it is accessible only via RL (Line 67).

Finally, at Line 69 it updates the pred and curr of opn $n_{i}$ in local log, $t x \log _{i}$. At Line 71 releases the locks on all the pred and curr in increasing order of keys to avoid deadlocks and return Commit.

We illustrate the helping methods of rv_method, t_insert(), and upd_method in detail as follows:

rv_Validation(): It is called by the rv_method, t_insert(), and upd_method. It identifies the conflicts among the concurrent methods of different transactions. Consider an example shown in Figure 9, where two concurrent conflicting methods of different transactions are working on the same key $k_{4}$. Initially, at stage $s_{1}$ in Figure 9 (c) both 


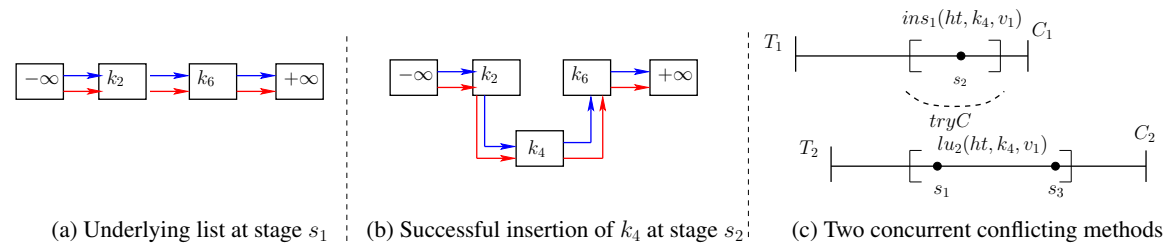

Figure 9: Illustration of rv_Validation()

the conflicting method optimistically (without acquiring locks) identify the same pred and curr keys for key $k_{4}$ from $B_{k}$.lazyrb-list in Figure 9 (a). At stage $s_{2}$ in Figure 9 (c), method $i n s_{1}\left(h t, k_{4}, v_{1}\right)$ of transaction $T_{1}$ acquired the lock on pred and curr keys and inserted the node into $B_{k}$.lazyrb-list as shown in Figure 9 (b). After successful insertion by $T_{1}$, pred and curr have been changed for $l u_{2}\left(h t, k_{4}\right)$ at stage $s_{3}$ in Figure 9 (c). So, the above modified information is delivered by $r v_{-}$Validation method at Line 74 when (preds $[0] . B L \neq \operatorname{currs}[1])$ for $l u_{2}\left(h t, k_{4}\right)$. After that again it will find the new pred and curr for $l u_{2}\left(h t, k_{4}, v_{1}\right)$ and eventually it will commit.

$\overline{\text { Algorithm } 5 r v \text { Validation(): Validate against the conflicting method of different trans- }}$ actions.

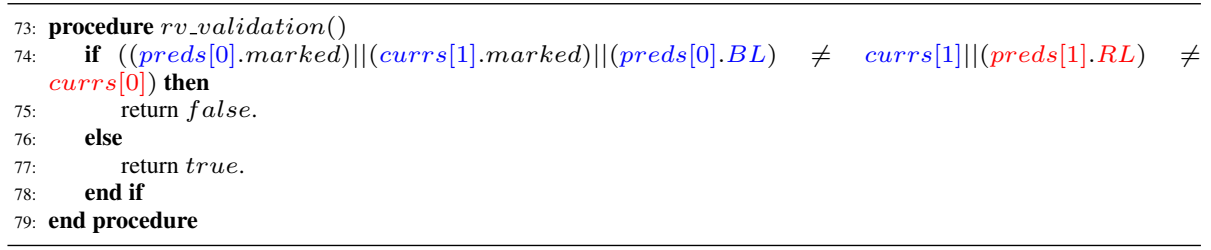

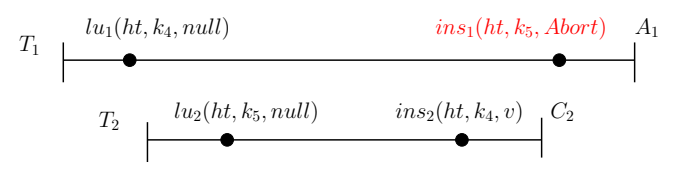

(a) Opaque history: $T_{1}$ Abort

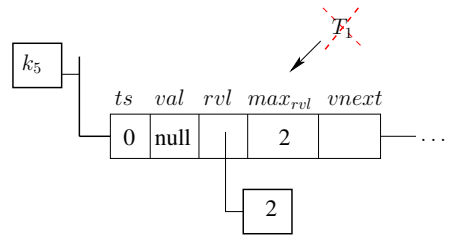

(b) Underlying Data structure(DS)

Figure 10: Illustration of tryC_Validation()

tryC_Validation(): It is called by t_insert(), and upd_method in $\operatorname{try} C$. First, it does the $r v$ Validation() in Line 81 If its successful and key $k$ exists in the $B_{k}$.lazyrb-list and let $n$ as a node of $k$. Then algorithm identifies the version of $n, v e r_{j}$ which has the 
timestamp $j$ such that $j$ has the largest timestamp smaller than $i$ (timestamp of $T_{i}$ ) at Line 85 If $\max _{r v l}$ of $v e r_{j}$ is greater than the timestamp of $i$ then the algorithm returns false (in Line 877 and eventually, return Abort in Line 38 or Line 53 . Consider an example as shown in Figure 10 (a), where second method $i n s_{1}\left(h t, k_{5}\right)$ of transaction $T_{1}$ returns Abort because higher timestamp of transaction $T_{2}$ is already present in the $\max _{r v l}$ of version $T_{0}$ identified by $T_{1}$ in Figure 10 (b).
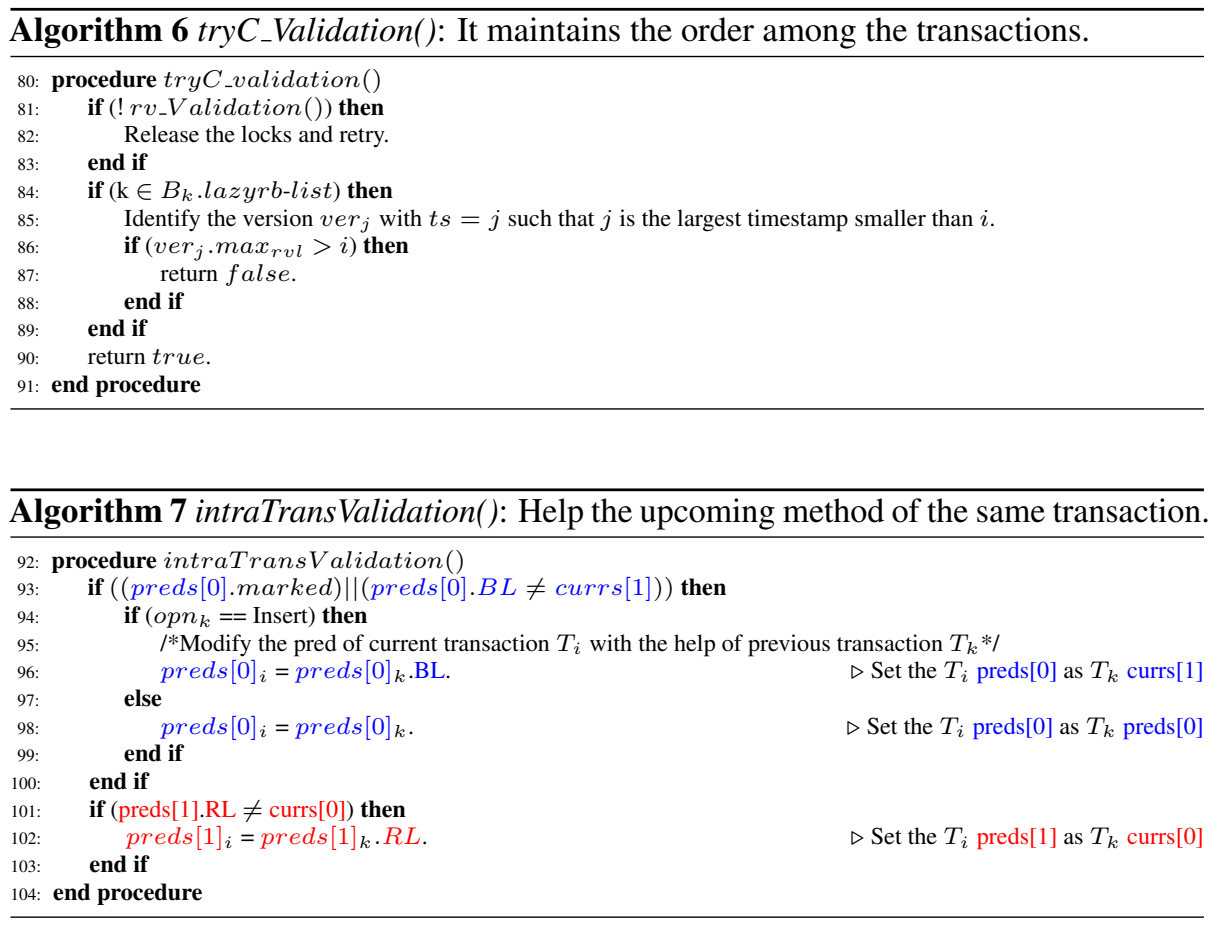

intraTransValidation(): It is called by upd_method in tryC. If two upd_methods of the same transaction have at least one common shared node among its recorded pred and curr keys, then the previous upd_method effect may overwrite if the current upd_method of pred and curr keys are not updated according to the updates done by the previous upd_method. Thus to solve this we have intraTransValidation() that modifies the pred and curr keys of current operation based on the previous operation from Line 93 to Line 103 . Consider an example as shown in Figure 11, where two upd_methods of transaction $T_{1}$ are $i n s_{11}\left(h t, k_{4}, v_{1}\right)$ and $i n s_{12}\left(h t, k_{6}, v_{2}\right)$ in Figure 11 (c). At stage $s_{1}$ in Figure 11 (c) both the upd_methods identify the same pred and curr 
from underlying DS as $B_{k}$.lazyrb-list shown in Figure 11 (a). After the successful insertion done by first upd_method at stage $s_{2}$ in Figure 11 (c), key $k_{4}$ is part of $B_{k}$.lazyrb-list (Figure 11(b)). At stage $s_{3}$ in Figure 11 (c), ins $s_{12}\left(h t, k_{6}, v_{2}\right)$ identified (preds $[0] . B L \neq$ currs $[1])$ in intraTransValidation() at Line 93 . So it updates the preds $[0]$ in Line 96 for correct updation in $B_{k}$.lazyrb-list.

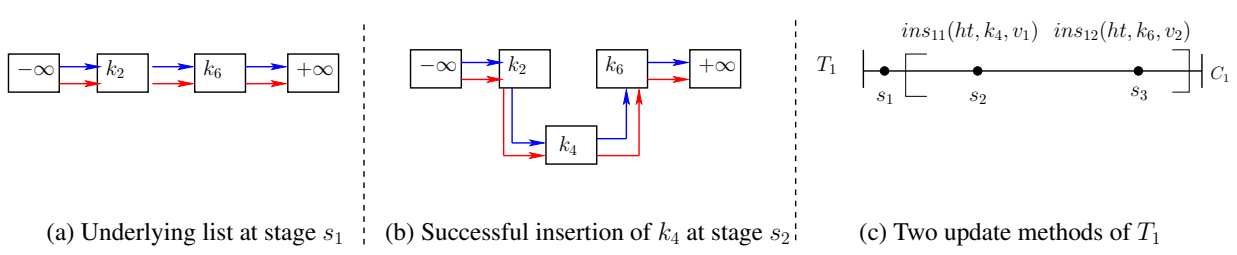

Figure 11: Illustration of intraTrans Validation()

\section{Correctness of OPT-MVOSTM}

In this section, we will prove that our implementation satisfies opacity. Consider the history $H$ generated by OPT-MVOSTM algorithm. Recall that only the $t$ _begin, rv_method, t_insert(), upd_method (or $\operatorname{try} C$ ) access shared memory.

Note that $H$ is not necessarily sequential: the transactional methods can execute in an overlapping manner. To reason about correctness, we have to prove $H$ is opaque. Since we defined opacity for histories which are sequential, we order all the overlapping methods in $H$ to get an equivalent sequential history. We then show that this resulting sequential history satisfies method.

We order overlapping methods of $H$ as follows: (1) two overlapping $t$ _begin methods based on the order in which they obtain lock over the counter; (2) two rv_methods accessing the same key $k$ by their order of unlocking over $\langle$ preds [0], preds [1], currs [0], currs $[1]\rangle$ of $k$; (3) an rv_method $\operatorname{rvm}_{i}(k)$ and a $t_{-}$insert $_{j}()$, of a transaction $T_{j}$ accessing the same key $k$, are ordered by their order of unlocking over $\langle$ preds[0], preds[1], currs[0], currs[1] $\rangle$ of $k$; (4) an rv_method $\operatorname{rvm}_{i}(k)$ and a $\operatorname{try} C_{j}$, of a transaction $T_{j}$ which has written to $k$, are similarly ordered by their order of unlocking over $\langle$ preds[0], preds[1], currs[0], currs[1]〉 of $k$; (5) two t_insert() methods accessing the same key $k$ by their order of unlocking over $\langle$ preds $[0]$, preds $[1]$, currs $[0]$, currs $[1]\rangle$ 
of $k$; (6) a $t_{-} i n s e r t_{i}()$ and a $\operatorname{try} C_{j}$, of a transaction $T_{j}$ which has written to $k$, are similarly ordered by their order of unlocking over $\langle$ preds [0], preds [1], currs $[0]$, currs $[1]\rangle$ of $k$; (7) similarly, two tryC methods based on the order in which they unlock over $\langle$ preds[0], preds[1], currs[0], currs[1]〉 of same key $k$.

Combining the real-time order of events with above-mentioned order, we obtain a partial order which we denote as $\operatorname{lockOrder}_{H}$. (It is a partial order since it does not order overlapping rv_methods on different keys or an overlapping rv_method and a tryC which do not access any common key).

In order for $H$ to be sequential, all its methods must be ordered. Let $\alpha$ be a total order or linearization of methods of $H$ such that when this order is applied to $H$, it is sequential. We denote the resulting history as $H^{\alpha}=$ linearize $(H, \alpha)$. We now argue about the validity of histories generated by the algorithm.

Lemma 5. Consider a history $H$ generated by the OPT-MVOSTM algorithm. Let $\alpha$ be a linearization of $H$ which respects lockOrder ${ }_{H}$, i.e. lockOrder lo $_{H} \subseteq$. Then $H^{\alpha}=$ linearize $(H, \alpha)$ is valid.

Proof: Consider a successful rv_method $r v m_{i}(k)$ that returns value $v$. The rv_method first obtains the lock on $\langle$ preds[0], preds[1], currs[0], currs[1]〉 of key $k$. Thus the value $v$ returned by the rv_method must have already been stored in $k$ 's version list by a transaction, say $T_{j}$ when it successfully returned $\mathrm{OK}$ from its $\operatorname{try} C$ method. For this to have occurred, $T_{j}$ must have successfully locked and released $\langle$ preds [0], preds [1], currs $[0]$, currs $[1]\rangle$ of $k$ prior to $T_{i}$ 's locking method. Thus from the definition of $\operatorname{lockOrder}_{H}$, we get that $\operatorname{try} C_{j}(o k)$ occurs before $\operatorname{rvm}_{i}(k, v)$ which also holds in $\alpha$.

It can be seen that for proving correctness, any linearization of a history $H$ is sufficient as long as the linearization respects lockOrder $_{H}$. The following lemma formalizes this intuition,

Lemma 6. Consider a history $H$. Let $\alpha$ and $\beta$ be two linearizations of $H$ such that both of them respect lockOrder ${ }_{H}$, i.e. lockOrder ${ }_{H} \subseteq \alpha$ and lockOrder ${ }_{H} \subseteq \beta$. Then, $H^{\alpha}=$ linearize $(H, \alpha)$ is opaque if $H^{\beta}=\operatorname{linearize}(H, \beta)$ is opaque.

Proof: From Lemma 5, we get that both $H^{\alpha}$ and $H^{\beta}$ are valid histories. Now let us consider each case 
If: Assume that $H^{\alpha}$ is opaque. Then, we get that there exists a legal t-sequential history $S$ that is equivalent to $\overline{H^{\alpha}}$. From the definition of $H^{\beta}$, we get that $\overline{H^{\alpha}}$ is equivalent to $\overline{H^{\beta}}$. Hence, $S$ is equivalent to $\overline{H^{\beta}}$ as well. We also have that, $\prec_{H^{\alpha}}^{R T} \subseteq \prec_{S}^{R T}$. From the definition of lockOrder ${ }_{H}$, we get that $\prec_{H^{\alpha}}^{R T}=\prec_{\text {lockOrder }_{H}}^{R T}=\prec_{H^{\beta}}^{R T}$. This automatically implies that $\prec_{H^{\beta}}^{R T} \subseteq \prec_{S}^{R T}$. Thus $H^{\beta}$ is opaque as well.

Only if: This proof comes from symmetry since $H^{\alpha}$ and $H^{\beta}$ are not distinguishable.

This lemma shows that, given a history $H$, it is enough to consider one sequential history $H^{\alpha}$ that respects lockOrder ${ }_{H}$ for proving correctness. If this history is opaque, then any other sequential history that respects $\operatorname{lockOrder}_{H}$ is also opaque.

Consider a history $H$ generated by OPT-MVOSTM algorithm. We then generate a sequential history that respects lockOrder ${ }_{H}$. For simplicity, we denote the resulting sequential history of OPT-MVOSTM as $H_{t o}$. Let $T_{i}$ be a committed transaction in $H_{t o}$ that writes to $k$ (i.e. it creates a new version of $k$ ).

To prove the correctness, we now introduce some more notations. We define $H_{t o} . s t l\left(T_{i}, k\right)$ as a committed transaction $T_{j}$ such that $T_{j}$ has the smallest timestamp larger (or stl) than $T_{i}$ in $H_{t o}$ that writes to $k$ in $H_{t o}$. Similarly, we define $H_{t o} . l t s\left(T_{i}, k\right)$ as a committed transaction $T_{k}$ such that $T_{k}$ has the largest timestamp smaller (or lts) than $T_{i}$ that writes to $k$ in $H_{t o}$. Using these notations, we describe the following properties and lemmas on $H_{t o}$,

Property 7. Every transaction $T_{i}$ is assigned a unique numeric timestamp $i$.

Property 8. If a transaction $T_{i}$ begins after another transaction $T_{j}$ then $j<i$.

Lemma 9. If a transaction $T_{k}$ looks up key $k_{x}$ from (a committed transaction) $T_{j}$ then $T_{j}$ is a committed transaction updating to $k_{x}$ with $j$ being the largest timestamp smaller than $k$. Formally, $T_{j}=H_{t o} . l t s\left(T_{k}, k_{x}\right)$.

Proof: We prove it by contradiction. So, assume that transaction $T_{k}$ looks up key $k_{x}$ from $T_{i}$ that has committed before $T_{j}$ so, from Property $8, i<k$ and $k<j$ i.e. $i$ is not largest timestamp smaller than $k$. But given statement in this lemma is $i<j<k$ which 
contradicts our assumption. Hence, $T_{k}$ looks up key $k_{x}$ from $T_{j}$ which is the largest timestamp smaller than $k$.

Lemma 10. Suppose a transaction $T_{k}$ looks up $k_{x}$ from (a committed transaction) $T_{j}$ in $H_{t o}$, i.e. $\left\{\operatorname{up}_{j}\left(k_{x, j}, v\right), \operatorname{rvm}_{k}\left(k_{x, i}, v\right)\right\} \in \operatorname{evts}\left(H_{t o}\right)$. Let $T_{i}$ be a committed transaction that updates to $k_{x}$, i.e. $\operatorname{up}_{i}\left(k_{x, i}, u\right) \in$ evts $\left(T_{i}\right)$. Then, the timestamp of $T_{i}$ is either less than $T_{j}$ 's timestamp or greater than $T_{k}$ 's timestamp, i.e. $i<j \oplus k<i$ (where $\oplus$ is XOR operator).

Proof: We will prove this by contradiction. Assume that $i<j \oplus k<i$ is not true. This implies that, $j<i<k$. But from the implementation of rv_method and try $C$ methods, we get that either transaction $T_{i}$ is aborted or $T_{k}$ looks up $k$ from $T_{i}$ in $H$. Since neither of them are true, we get that $j<i<k$ is not possible. Hence, $i<j \oplus k<i$.

To show that $H_{t o}$ satisfies opacity, we use the graph characterization developed above in Section 3. For the graph characterization, we use the version order defined using timestamps. Consider two committed transactions $T_{i}, T_{j}$ such that $i<j$. Suppose both the transactions write to key $k$. Then the versions created are ordered as $k_{i} \ll k_{j}$. We denote this version order on all the keys created as $\ll_{t o}$. Now consider the opacity graph of $H_{t o}$ with version order as defined by $\ll_{t o}, G_{t o}=O P G\left(H_{t o}, \ll_{t o}\right)$. In the following lemmas, we will prove that $G_{t o}$ is acyclic.

Lemma 11. All the edges in $G_{t o}=O P G\left(H_{t o}, \ll_{t o}\right)$ are in timestamp order, i.e. if there is an edge from $T_{j}$ to $T_{i}$ then the $j<i$.

Proof: To prove this, let us analyze the edges one by one,

- rt edges: If there is an rt edge from $T_{j}$ to $T_{i}$, then $T_{j}$ terminated before $T_{i}$ started. Hence, from Property 8 we get that $j<i$.

- rvf edges: This follows directly from Lemma9

- mv edges: The mv edges relate a committed transaction $T_{k}$ updates to a key $k, u p_{k}(k, v)$; a successful rv_method $r v m_{j}(k, u)$ belonging to a transaction $T_{j}$ looks up $k$ updated by a committed transaction $T_{i}, u p_{i}(k, u)$. Transactions $T_{i}, T_{k}$ 
create new versions $k_{i}, k_{k}$ respectively. According to $\ll_{t o}$, if $k_{k} \ll_{t o} k_{i}$, then there is an edge from $T_{k}$ to $T_{i}$. From the definition of $\ll_{t o}$ this automatically implies that $k<i$.

On the other hand, if $k_{i} \ll_{t o} k_{k}$ then there is an edge from $T_{j}$ to $T_{k}$. Thus, in this case, we get that $i<k$. Combining this with Lemma 10, we get that $j<k$.

Thus in all the cases, we have shown that if there is an edge from $T_{j}$ to $T_{i}$ then the $j<i$.

Theorem 12. Any history $H_{\text {to }}$ generated by OPT-MVOSTM is opaque.

Proof: From the definition of $H_{t o}$ and Lemma 5, we get that $H_{t o}$ is valid. We show that $G_{t o}=O P G\left(H_{t o}, \ll_{t o}\right)$ is acyclic. We prove this by contradiction. Assume that $G_{t o}$ contains a cycle of the form, $T_{c 1} \rightarrow T_{c 2} \rightarrow . . T_{c m} \rightarrow T_{c 1}$. From Lemma11 we get that, $c 1<c 2<\ldots<c m<c 1$ which implies that $c 1<c 1$. Hence, a contradiction. This implies that $G_{t o}$ is acyclic. Thus from Theorem 4, we get that $H_{t o}$ is opaque.

Now, it is left to show that our algorithm is live, i.e., under certain conditions, every operation eventually completes. We have to show that the transactions do not deadlock. This is because all the transactions lock all $\langle$ preds[0],preds[1], currs[0], currs [1]〉 of keys in a predefined order. As discussed earlier, the STM system orders all $\langle$ preds[0], preds[1], currs [0], currs[1]〉 of keys. We denote this order as accessOrderand denote it as $\prec_{a o}$. Thus $k_{1} \prec_{a o} k_{2} \prec_{a o} \ldots \prec_{a o} k_{n}$.

From accessOrder, we get the following property

Property 13. Suppose transaction $T_{i}$ accesses shared objects $p$ and $q$ in $H$. If $p$ is ordered before $q$ in accessOrder, then lock $(p)$ by transaction $T_{i}$ occurs before lock $(q)$. Formally, $\left(p \prec_{a o} q\right) \Leftrightarrow\left(\operatorname{lock}(p)<_{H} \operatorname{lock}(q)\right)$.

Theorem 14. OPT-MVOSTM with unbounded versions ensures that $r v \_m e t h o d s$ do not abort.

Proof: This is self-explanatory with the help of OPT-MVOSTM algorithm because each key is maintaining multiple versions in the case of unbounded versions. So rv_method always finds a correct version to read it from. Thus, rv_methods do not abort. 


\section{Experimental Evaluation}

This section describes the experimental analysis of proposed OPT-MVOSTMs with state-of-the-art STMs. We have three main goals in this section: (1) Analyze the performance benefits of the optimized multi-version object based STMs (or $O P T$ MVOSTMS) over multi-version object based STMs (or MVOSTMs). (2) Evaluate the benefit of OPT-MVOSTMS over the single-version object based STMs (or OSTMs), and (3) Analyze the benefit of OPT-MVOSTMS over multi-version read-write STMs. We implement hash-table object and list object as OPT-HT-MVOSTM and OPT-listMVOSTM described in Section 5 We also consider the extension of this optimized multi-version object STMs to reduce memory usage. Specifically, we consider a variant that implements garbage collection with unbounded versions and another variant where the number of versions never exceeds a given threshold $K$ for both OPT-HT-MVOSTMs and $O P T$-list-MVOSTMs.

Experimental system: The Experimental system is a large-scale 2-socket Intel(R) Xeon(R) CPU E5-2690 v4 @ 2.60GHz with 14 cores per socket and two hyper-threads (HTs) per core, for a total of 56 threads. Each core has a private 32KB L1 cache and $256 \mathrm{~KB} \mathrm{~L} 2$ cache (which is shared among HTs on that core). All cores on a socket share a 35MB L3 cache. The machine has 32GB of RAM and runs Ubuntu 16.04.2 LTS. All code was compiled with the GNU C++ compiler (G++) 5.4.0 with the build target x86_64-Linux-gnu and compilation option - std=c++1x -O3.

STM implementations: We have taken the implementation of NOrec-list [3], Boostinglist [4], Trans-list [16], ESTM [2], and RWSTM directly from the TLDS framework And the implementation of MVOSTM [1], OSTM [6] and MVTO [10] from our PDCRL library We implemented our algorithms in C++. Each STM algorithm first creates $\mathrm{N}$-threads, each thread, in turn, spawns a transaction. Each transaction exports $t$ _begin, t_insert, $t$ _lookup, t_delete and tryC methods as described in Section 2

Methodology:5 We have considered three types of workloads: (W1) Li - Lookup

\footnotetext{
${ }^{3}$ https://ucf-cs.github.io/tlds/

${ }^{4}$ https://github.com/PDCRL/

${ }^{5}$ Code is available here: https://github.com/PDCRL/MVOSTM/OPT-MVOSTM
} 
intensive (90\% lookup, $8 \%$ insert, and 2\% delete), (W2) $\mathrm{Mi}$ - Mid intensive (50\% lookup, 25\% insert, and 25\% delete), and (W3) Ui - Update intensive (10\% lookup, $45 \%$ insert, and $45 \%$ delete). The experiments are conducted by varying number of threads from 2 to 64 in power of 2, with 1000 keys randomly chosen. We assume that the hash-table of OPT-HT-MVOSTM has five buckets and each of the bucket (or list in case of OPT-list-MVOSTM) can have a maximum size of 1000 keys. Each transaction, in turn, executes 10 operations which include $t_{-}$lookup, t_delete, and $t_{-} i n s e r t$ operations. We take an average over 10 results as the final result for each experiment.

Results: Figure 12 represents the performance benefit of all the variants of proposed optimized MVOSTM with all variants of MVOSTM for hash-table objects. It shows OPT-HT-KOSTM performs best among all the algorithms (OPT-HT-MVOSTM-GC, OPT-HT-MVOSTM, HT-KOSTM, HT-MVOSTM-GC, HT-MVOSTM) by a factor of 1.02, 1.11, 1.05, 1.07, 1.22 for workload W1, 1.06, 1.09, 1.07, 1.08, 1.15 for workload $\mathrm{W} 2$, and 1.01, 1.03, 1.02, 1.03, 1.08 for workload W3 respectively. Along with this, Figure 13 shows the abort count respective algorithms on workload W1, W2, and W3. This represents for less number of threads, the number of aborts are almost same for all the algorithms. But while increasing the number of threads, the number of aborts are least in OPT-HT-KOSTM as compare to others. So, we compare the performance of OPTHT-KOSTM with the state-of-the-art STMs as shown in Figure 14 OPT-HT-KOSTM outperforms all the algorithms (HT-OSTM, ESTM, RWSTM, HT-MVTO, HT-KSTM) by a factor of 3.62, 3.95, 3.44, 2.75, 1.85 for $\mathrm{W} 1,1.44,2.36,4.45,9.84,7.42$ for $\mathrm{W} 2$, and $2.11,4.05,7.84,12.94,10.70$ for $\mathrm{W} 3$ respectively. The corresponding number of aborts are represented in Figure 15. Number of aborts are minimum for OPT-HTKOSTM as compare to other state-of-the-art STMs. Especially, the number of aborts for OPT-HT-KOSTM is almost negligible as compared to HT-OSTM on lookup-intensive workload (W1) because OPT-HT-KOSTM finds a correct version to looks up as shown in Figure 15 (a).

The observation of optimized list based MVOSTM is similar as optimized hash-table based MVOSTM. Figure 16 represents the performance benefit of all the variants of proposed optimized MVOSTM with all variants of MVOSTM for list objects. It shows 
OPT-list-KOSTM performs best among all the algorithms (OPT-list-MVOSTM-GC, OPT-list-MVOSTM, list-KOSTM, list-MVOSTM-GC, list-MVOSTM) by a factor of $1.14,1.24,1.21,1.20,1.35$ for $\mathrm{W} 1,1.06,1.07,1.12,1.13,1.20$ for $\mathrm{W} 2$, and 1.09, 1.19, $1.11,1.17,1.31$ for $\mathrm{W} 3$ respectively. Along with this, Figure 17 shows the minimum abort count by OPT-list-KOSTM as compare to other algorithms on workload W1, W2, and W3. Hence, we choose the best-proposed algorithm OPT-list-KOSTM and compare with the state-of-the-art list based STMs.

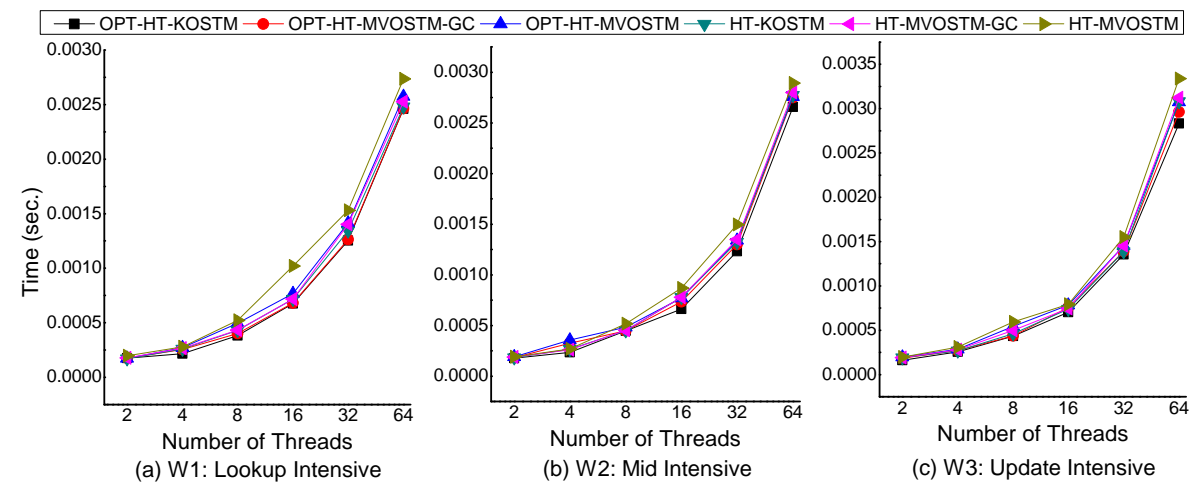

Figure 12: Time comparison among variants of OPT-HT-MVOSTMs and HT-MVOSTMs on hash-table

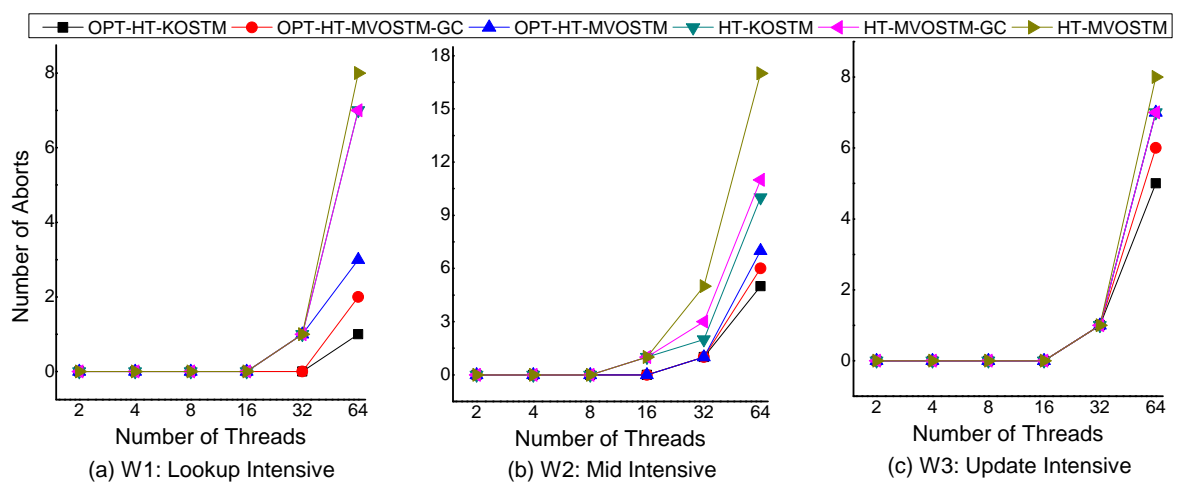

Figure 13: Abort count among variants of OPT-HT-MVOSTMS and HT-MVOSTMs on hash-table

Figure 18 represents OPT-list-KOSTM outperforms all the algorithms (list-OSTM, Trans-list, Boosting-list, NOrec-list, list-MVTO, list-KSTM) by a factor of 2.56, 25.38, 23.57, 27.44, 13.34, 5.99 for $\mathrm{W} 1,1.51,20.54,24.27,29.45,24.89,19.78$ for $\mathrm{W} 2$, and 2.91, 32.88, 28.45, 40.89, 173.92, 124.89 for W3 respectively. Similarly, Figure 19 depicts that $O P T$-list-KOSTM obtained the least number of aborts as compare to others 
on the respective workloads.

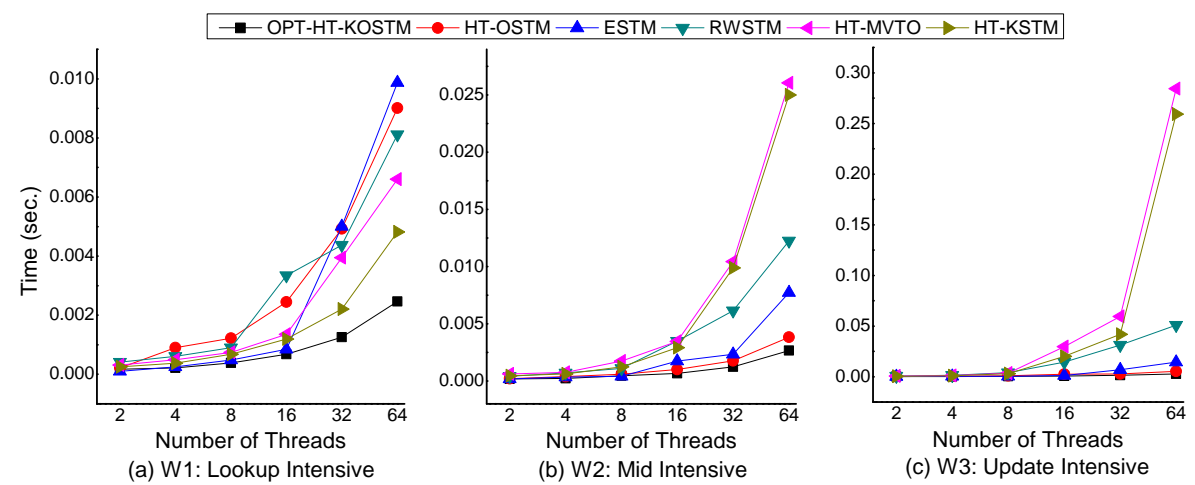

Figure 14: Time comparison of OPT-HT-KOSTM and State-of-the-art hash-table based STMs

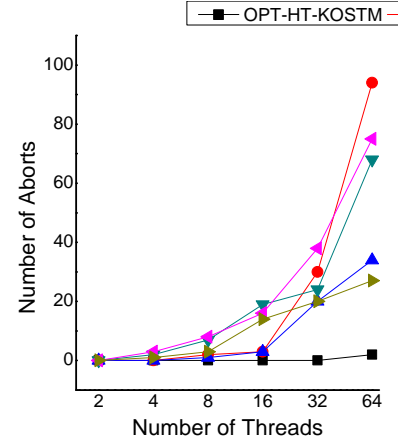

(a) W1: Lookup Intensive

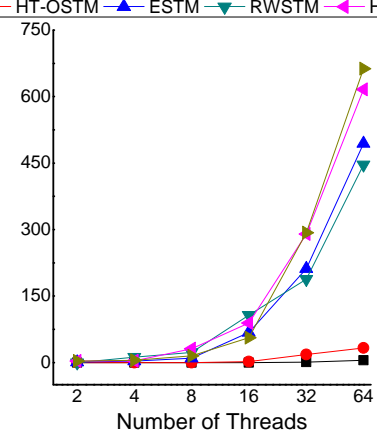

(b) W2: Mid Intensive

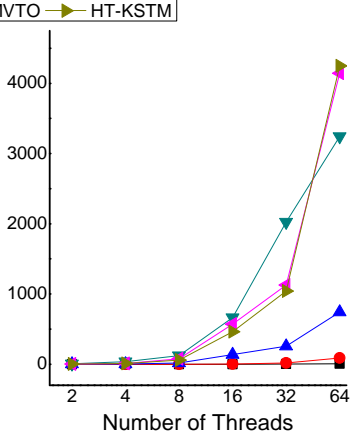

(c) W3: Update Intensive

Figure 15: Abort count of OPT-HT-KOSTM and State-of-the-art hash-table based STMs

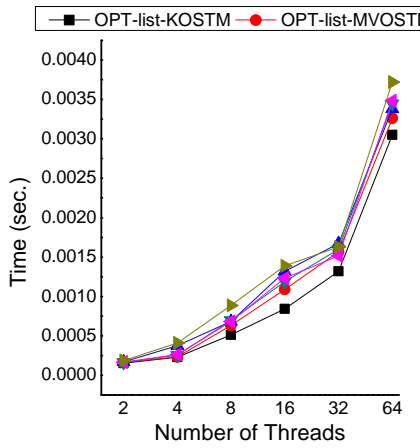

(a) W1: Lookup Intensive

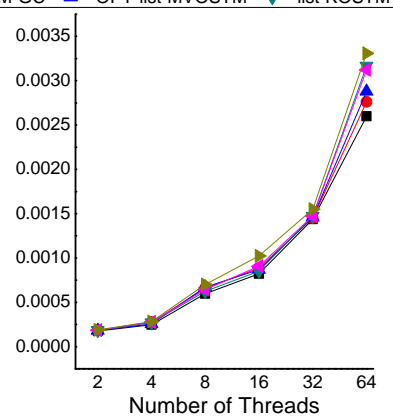

(b) W2: Mid Intensive

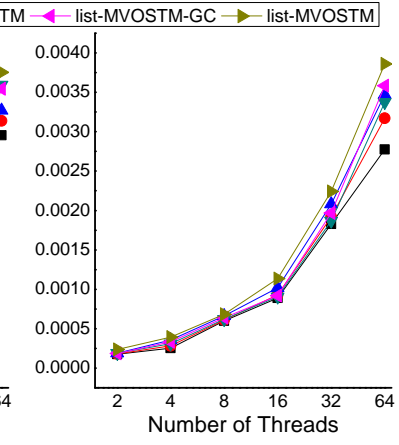

(c) W3: Update Intensive

Figure 16: Time comparison among variants of OPT-list-MVOSTMs and list-MVOSTMs on list 


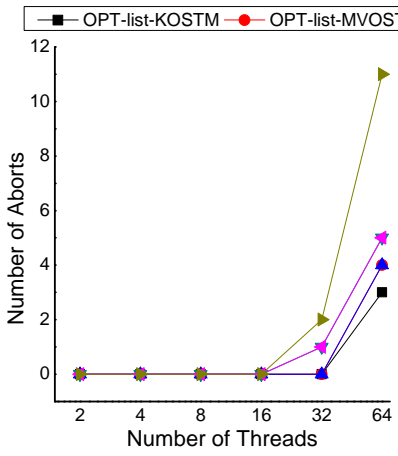

(a) W1: Lookup Intensive

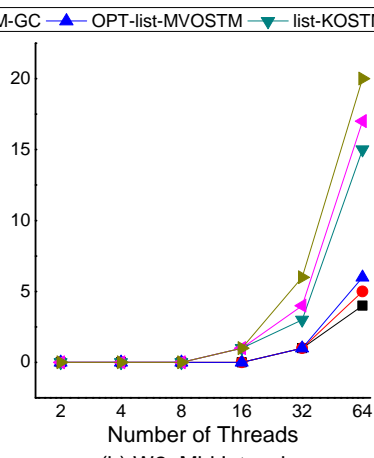

(b) W2: Mid Intensive

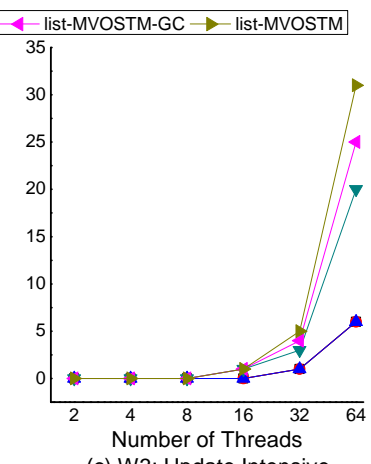

(c) W3: Update Intensive

Figure 17: Abort count among variants of OPT-list-MVOSTMs and list-MVOSTMs on list

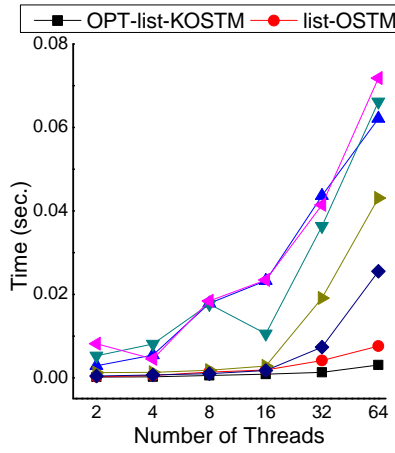

(a) W1: Lookup Intensive

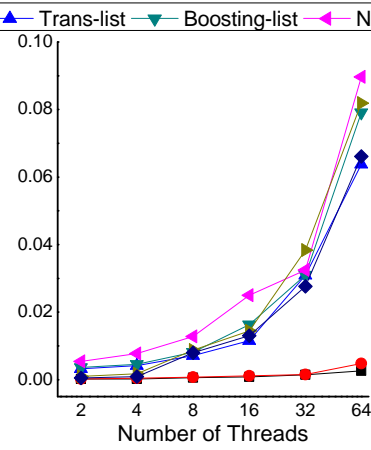

(b) W2: Mid Intensive

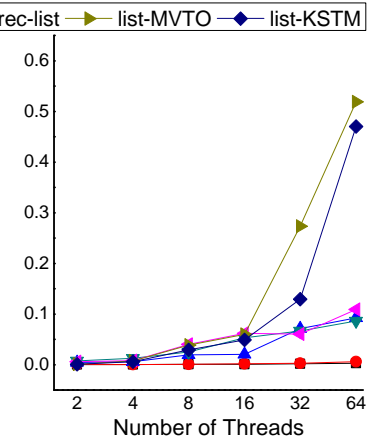

(c) W3: Update Intensive

Figure 18: Time comparison of OPT-list-KOSTM and State-of-the-art list based STMs

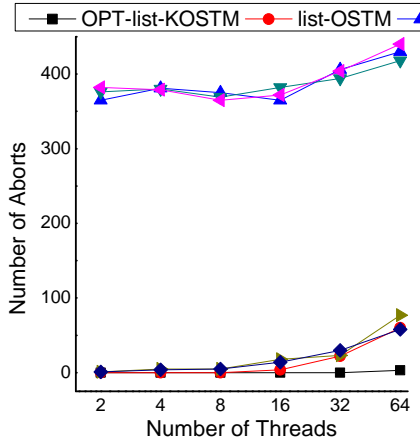

(a) W1: Lookup Intensive

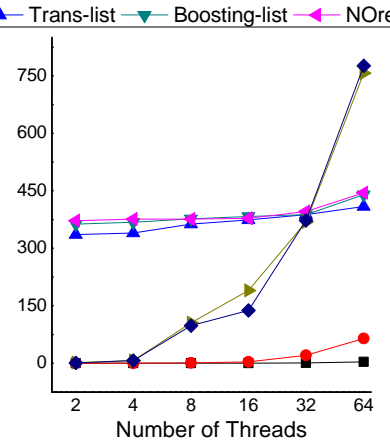

(b) W2: Mid Intensive

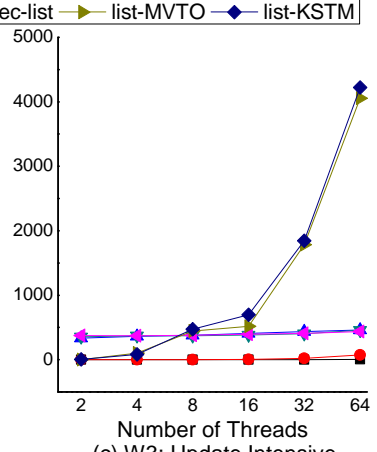

(c) W3: Update Intensive

Figure 19: Abort count of OPT-list-KOSTM and State-of-the-art list based STMs 


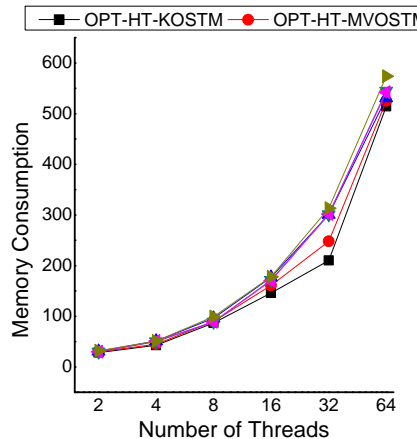

(a) W1: Lookup Intensive

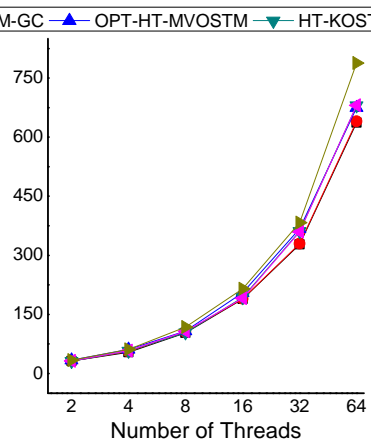

(b) W2: Mid Intensive

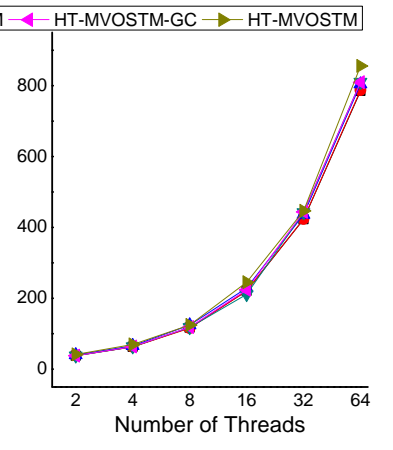

(c) W3: Update Intensive

Figure 20: Memory consumption among variants of OPT-HT-MVOSTMs and HT-MVOSTMs on hash-table

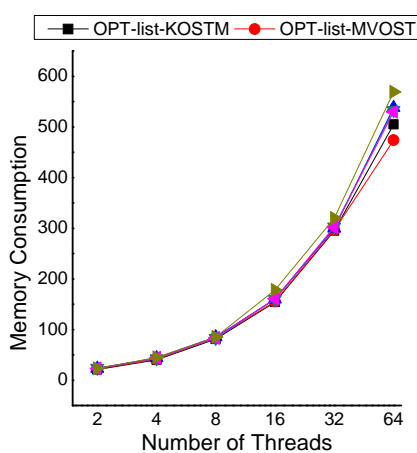

(a) W1: Lookup Intensive

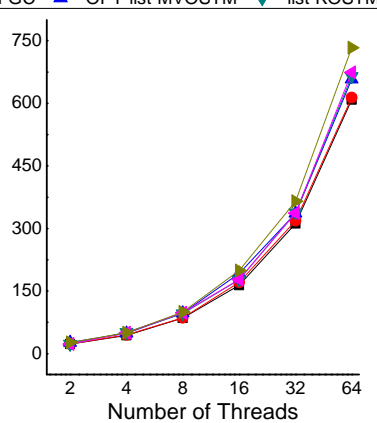

(b) W2: Mid Intensive

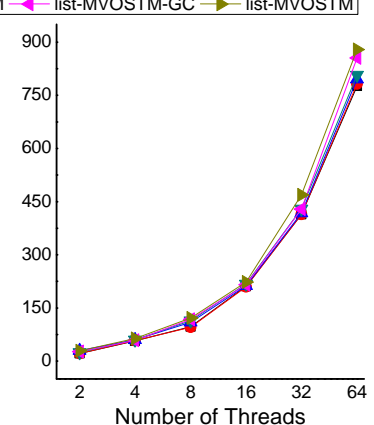

(c) W3: Update Intensive

Figure 21: Memory consumption among variants of OPT-list-MVOSTMs and list-MVOSTMs on list

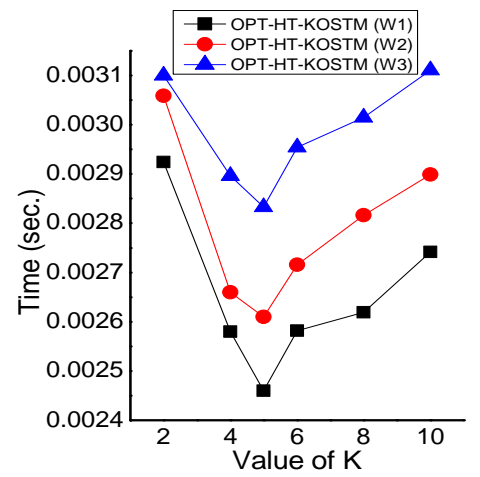

(a) OPT-HT-KOSTM

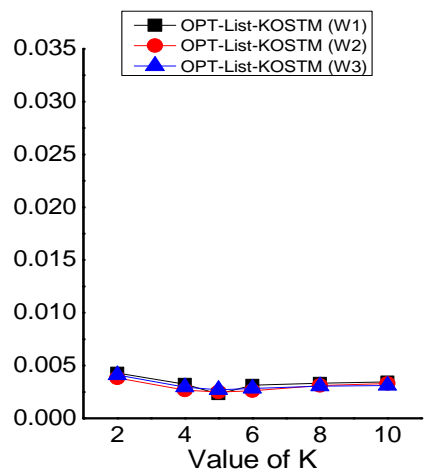

(b) OPT-List-KOSTM

Figure 22: Optimal Value of $K$ for OPT-HT-KOSTM and OPT-list-KOSTM 
As explained in Section 5, for efficient memory utilization, we develop two variations of OPT-MVOSTM. The first, OPT-MVOSTM-GC, uses unbounded versions but performs garbage collection. This is achieved by deleting non-latest versions whose timestamp is less than the timestamp of the least live transaction. OPT-MVOSTM-GC gave a performance gain of $16 \%$ over OPT-MVOSTM without garbage collection in the best case which is on workload W1 with 64 number of threads. We did one more optimization in OPT-MVOSTM-GC on the marked node exist in the RL to make it search efficiently. This is achieved by deleting a marked node from RL whose $\max _{r v l}$ of the last version is less than the timestamp of the least live transaction. The second, OPT-KOSTM, keeps at most $K$ versions by replacing the oldest version when $(K+1)^{t h}$ version is created by a current transaction as explained in Section 5 , OPT-KOSTM shows a performance gain of $24 \%$ over OPT-MVOSTM without garbage collection in the best case which is on workload W1 with 64 number of threads. As OPT-KOSTM has a limited number of versions while $O P T-M V O S T M-G C$ can have infinite versions, the memory consumed by $O P T-K O S T M$ is also less than OPT-MVOSTM-GC. We have integrated these variations in both hash-table based (OPT-HT-MVOSTM-GC and OPT-HT-KOSTM) and linked-list based MVOSTMs (OPT-list-MVOSTM-GC and OPT-list-KOSTM), we observed that these two variations increase the performance, concurrency and reduce the number of aborts as compared to OPT-MVOSTM which does not perform garbage collection.

Memory Consumption by OPT-MVOSTM-GC and OPT-KOSTM: As depicted above OPT-KOSTM performs better than OPT-MVOSTM-GC. Continuing the comparison between the two variations of OPT-MVOSTM we chose another parameter as memory consumption. Here we test for the memory consumed by each variation algorithms in creating a version of a key. We count the total versions created, where creating a version increases the counter value by 1 and deleting a version decreases the counter value by 1 . Figure 20 depicts the comparison of memory consumption by all the variants of proposed optimized MVOSTM with all variants of MVOSTM for hash-table objects. OPT-HT-KOSTM consumes minimum memory among all the algorithms (OPT-HT-MVOSTM-GC, OPT-HT-MVOSTM, HT-KOSTM, HT-MVOSTM-GC, HT-MVOSTM) by a factor of 1.07, 1.16, 1.15, 1.15, 1.21 for $\mathrm{W} 1,1.01,1.08,1.06$, 
1.07, 1.19 for W2, and 1.01, 1.03, 1.02, 1.03, 1.08 for W3 respectively. Similarly, Figure 21 depicts the comparison of memory consumption by all the variants of proposed optimized MVOSTM with all variants of MVOSTM for list objects. OPT-list-KOSTM consumes minimum memory among all the algorithms (OPT-list-MVOSTM-GC, OPTlist-MVOSTM, list-KOSTM, list-MVOSTM-GC, list-MVOSTM) by a factor of 1.01, $1.05,1.05,1.04,1.11$ for $\mathrm{W} 1,1.02,1.1,1.1,1.111 .19$ for $\mathrm{W} 2$, and 1.01, 1.03, 1.05, $1.08,1.13$ for $\mathrm{W} 3$ respectively.

Finite version OPT-MVOSTM (OPT-KOSTM): To find the ideal value of $K$ such that performance as compared to OPT-MVOSTM-GC does not degrade or can be increased, we perform experiments on all the workloads (W1, W2, and W3) for both (OPT-HT-KOSTM and OPT-list-KOSTM). Figure 22 (a) and (b) shows the best value of $K$ as 5 for OPT-HT-KOSTM and OPT-list-KOSTM on all the workloads for both hash-table and list objects.

\section{Conclusion}

With the rise of multi-core systems, concurrent programming becomes popular. Concurrent programming using multiple threads has become necessary to utilize all the cores present in the system effectively. But concurrent programming is usually challenging due to synchronization issues between the threads.

In the past few years, several STMs have been proposed which address these synchronization issues and provide greater concurrency. STMs hide the synchronization and communication difficulties among the multiple threads from the programmer while ensuring correctness and hence making programming easy. Another advantage of STMs is that they facilitate compositionality of concurrent programs with great ease. Different concurrent operations that need to be composed to form a single atomic unit is achieved by encapsulating them in a single transaction.

In literature, most of the STMs are RWSTMs which export read and write operations. To improve the performance, a few researchers have proposed OSTMs [4, 5, 6] which export higher level objects operation such as hash-table insert, delete, and lookup etc. By leveraging the semantics of these higher level operations, these STMs provide greater 
concurrency. On the other hand, it has been observed in STMs and databases that by storing multiple versions for each t-object in case of RWSTMs provides greater concurrency [17, 10].

This paper proposed the notion of the optimized multi-version object based STMs (OPT-MVOSTMs) and compares their effectiveness with multi-version object based STMs (MVOSTMs), single-version object based STMs and multi-version read-write STMs. We find that OPT-MVOSTM provides a significant benefit over above-mentioned state-of-the-art STMs for different types of workloads. Specifically, we have evaluated the effectiveness of OPT-MVOSTM for the hash-table and list data structure as $O P T$ HT-MVOSTM and OPT-list-MVOSTM respectively.

OPT-HT-MVOSTM and OPT-list-MVOSTM use the unbounded number of versions for each key. To utilize the memory efficiently, we limit the number of versions and develop two variants for both hash-table and list data structures: (1) A garbage collection method in OPT-MVOSTM to delete the unwanted versions of a key, denoted as OPT-MVOSTM-GC. (2) Placing a limit of $K$ on the number of versions in $O P T$ MVOSTM, resulting in OPT-KOSTM. Both these variants (OPT-MVOSTM-GC and OPT-KOSTM) gave a performance gain of over $16 \%$ and $24 \%$ over OPT-MVOSTM in the best case. OPT-KOSTM consumes minimum memory among all the variants of it. We represent OPT-MVOSTM-GC in hash-table and list as OPT-HT-MVOSTM-GC and OPT-list-MVOSTM-GC respectively. Similarly, We represent OPT-KOSTM in hash-table and list as OPT-HT-KOSTM and OPT-list-KOSTM respectively.

OPT-HT-KOSTM performs best among its variants and outperforms state-of-the-art hash-table based STMs (HT-OSTM, ESTM, RWSTM, HT-MVTO, HT-KSTM) by a factor of $3.62,3.95,3.44,2.75,1.85$ for workload W1, 1.44, 2.36, 4.45, 9.84, 7.42 for workload W2, and 2.11, 4.05, 7.84, 12.94, 10.70 for workload W3 respectively. Similarly, OPT-list-KOSTM performs best among its variants and outperforms state-ofthe-art list based STMs (list-OSTM, Trans-list, Boosting-list, NOrec-list, list-MVTO, list-KSTM) by a factor of 2.56, 25.38, 23.57, 27.44, 13.34, 5.99 for W1, 1.51, 20.54, $24.27,29.45,24.89,19.78$ for $\mathrm{W} 2$, and $2.91,32.88,28.45,40.89,173.92,124.89$ for W3 respectively. We rigorously proved that $O P T-M V O S T M$ s satisfy the correctness criteria as opacity. 


\section{References}

[1] C. Juyal, S. S. Kulkarni, S. Kumari, S. Peri, A. Somani, An innovative approach to achieve compositionality efficiently using multi-version object based transactional systems, in: Stabilization, Safety, and Security of Distributed Systems - 20th International Symposium, SSS 2018, Tokyo, Japan, November 4-7, 2018, Proceedings, 2018, pp. 284-300. doi:10.1007/978-3-030-03232-6\_19. URL https://doi.org/10.1007/978-3-030-03232-6_19

[2] P. Felber, V. Gramoli, R. Guerraoui, Elastic Transactions, J. Parallel Distrib. Comput. 100 (C) (2017) 103-127. doi:10.1016/j.jpdc.2016.10.010, URL https://doi.org/10.1016/j.jpdc.2016.10.010

[3] L. Dalessandro, M. F. Spear, M. L. Scott, NOrec: Streamlining STM by Abolishing Ownership Records, in: R. Govindarajan, D. A. Padua, M. W. Hall (Eds.), PPOPP, ACM, 2010, pp. 67-78.

URL http://dblp.uni-trier.de/db/conf/ppopp/ppopp2010. html\#Dalessandross10

[4] M. Herlihy, E. Koskinen, Transactional boosting: a methodology for highlyconcurrent transactional objects, in: Proceedings of the 13th ACM SIGPLAN Symposium on Principles and Practice of Parallel Programming, PPOPP 2008, Salt Lake City, UT, USA, February 20-23, 2008, 2008, pp. 207-216. doi: $10.1145 / 1345206.1345237$ URL http://doi.acm.org/10.1145/1345206.1345237

[5] A. Hassan, R. Palmieri, B. Ravindran, Optimistic transactional boosting, in: Proceedings of the 19th ACM SIGPLAN Symposium on Principles and Practice of Parallel Programming, PPoPP '14, ACM, New York, NY, USA, 2014, pp. 387-388. doi:10.1145/2555243.2555283

URL http://doi.acm.org/10.1145/2555243.2555283

[6] S. Peri, A. Singh, A. Somani, Efficient means of achieving composability using object based semantics in transactional memory systems, in: Networked Systems - 6th International Conference, NETYS 2018, Essaouira, Morocco, May 
9-11, 2018, Revised Selected Papers, 2018, pp. 157-174. doi:10.1007/ 978-3-030-05529-5\_11.

URL https://doi.org/10.1007/978-3-030-05529-5_11

[7] S. Heller, M. Herlihy, V. Luchangco, M. Moir, W. N. S. III, N. Shavit, A Lazy Concurrent List-Based Set Algorithm, Parallel Processing Letters 17 (4) (2007) $411-424$.

[8] R. Guerraoui, M. Kapalka, On the correctness of transactional memory, in: Proceedings of the 13th ACM SIGPLAN Symposium on Principles and Practice of Parallel Programming, PPoPP '08, ACM, New York, NY, USA, 2008, pp. 175-184. doi:10.1145/1345206.1345233, URL http://doi.acm.org/10.1145/1345206.1345233

[9] G. Weikum, G. Vossen, Transactional Information Systems: Theory, Algorithms, and the Practice of Concurrency Control and Recovery, Morgan Kaufmann, 2002.

[10] P. Kumar, S. Peri, K. Vidyasankar, A timestamp based multi-version stm algorithm, in: Proceedings of the 15th International Conference on Distributed Computing and Networking - Volume 8314, ICDCN 2014, Springer-Verlag New York, Inc., New York, NY, USA, 2014, pp. 212-226. doi:10.1007/ 978-3-642-45249-9_14.

URL http://dx.doi.org/10.1007/978-3-642-45249-9_14

[11] R. Guerraoui, M. Kapalka, Principles of Transactional Memory, Synthesis Lectures on Distributed Computing Theory, Morgan \& Claypool Publishers, 2010. doi: 10.2200/S00253ED1V01Y201009DCT004.

URL https://doi.org/10.2200/S00253ED1V01Y201009DCT004

[12] P. Kuznetsov, S. Peri, Non-interference and local correctness in transactional memory, Theor. Comput. Sci. 688 (2017) 103-116. doi:10.1016/j.tcs. 2016.06.021.

URL https://doi.org/10.1016/j.tcs.2016.06.021 
[13] P. Kuznetsov, S. Ravi, On the cost of concurrency in transactional memory, in: Principles of Distributed Systems - 15th International Conference, OPODIS 2011, Toulouse, France, December 13-16, 2011. Proceedings, 2011, pp. 112-127. doi: $10.1007 / 978-3-642-25873-2 \backslash \_9$ URL https://doi.org/10.1007/978-3-642-25873-2_9

[14] C. H. Papadimitriou, The serializability of concurrent database updates J. ACM 26 (4) (1979) 631-653. doi:10.1145/322154.322158. URL http://doi.acm.org/10.1145/322154.322158

[15] T. L. Harris, A pragmatic implementation of non-blocking linked-lists, in: Proceedings of the 15th International Conference on Distributed Computing, DISC '01, Springer-Verlag, London, UK, UK, 2001, pp. 300-314. URL http://dl.acm.org/citation.cfm?id=645958.676105

[16] D. Zhang, D. Dechev, Lock-free transactions without rollbacks for linked data structures, in: Proceedings of the 28th ACM Symposium on Parallelism in Algorithms and Architectures, SPAA '16, ACM, New York, NY, USA, 2016, pp. 325-336. doi:10.1145/2935764.2935780.

URL http://doi.acm.org/10.1145/2935764.2935780

[17] D. Perelman, R. Fan, I. Keidar, On maintaining multiple versions in stm, in: Proceedings of the 29th ACM SIGACT-SIGOPS Symposium on Principles of Distributed Computing, PODC '10, ACM, New York, NY, USA, 2010, pp. 16-25. doi:10.1145/1835698.1835704

URL http://doi.acm.org/10.1145/1835698.1835704 